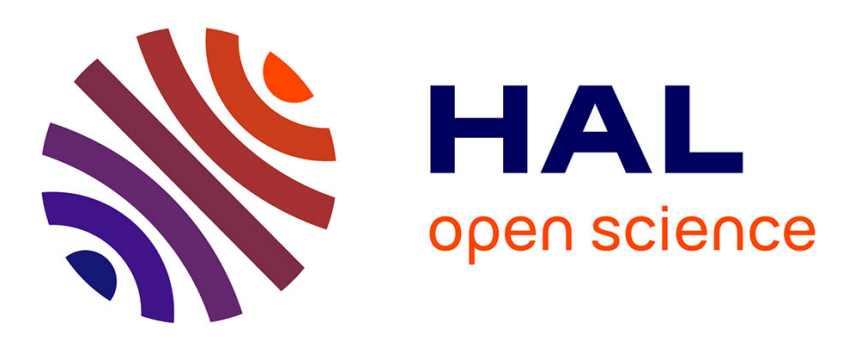

\title{
Arc-flow approach for single batch-processing machine scheduling
}

Renan Spencer Trindade, Olinto César Bassi de Araújo, Marcia Fampa

\section{To cite this version:}

Renan Spencer Trindade, Olinto César Bassi de Araújo, Marcia Fampa. Arc-flow approach for single batch-processing machine scheduling. Computers and Operations Research, 2021, 134, pp.105394. 10.1016/j.cor.2021.105394 . hal-03234783

\section{HAL Id: hal-03234783 \\ https://hal.science/hal-03234783}

Submitted on 3 Dec 2021

HAL is a multi-disciplinary open access archive for the deposit and dissemination of scientific research documents, whether they are published or not. The documents may come from teaching and research institutions in France or abroad, or from public or private research centers.
L'archive ouverte pluridisciplinaire HAL, est destinée au dépôt et à la diffusion de documents scientifiques de niveau recherche, publiés ou non, émanant des établissements d'enseignement et de recherche français ou étrangers, des laboratoires publics ou privés. 


\title{
Arc-flow approach for single batch-processing machine scheduling
}

\author{
Renan Spencer Trindade*1, Olinto C. B. de Araújo ${ }^{2}$, and Marcia Fampa ${ }^{3}$ \\ ${ }^{1}$ LIX, CNRS, École Polytechnique, Institut Polytechnique de Paris \\ ${ }^{2}$ Colégio Técnico Industrial de Santa Maria, Universidade Federal de Santa Maria \\ ${ }^{3}$ Instituto de Matemática, COPPE, Universidade Federal do Rio de Janeiro
}

\begin{abstract}
We address the problem of scheduling jobs with non-identical sizes and distinct processing times on a single batch-processing machine, aiming at minimizing the makespan. The extensive literature on this NP-hard problem mostly focuses on heuristics. Using an arc-flow based optimization approach, we construct a novel formulation that represents it as a problem of determining flows in graphs. The size of the formulation increases with the machine capacity and with the number of distinct sizes and processing times among the jobs, but it does not increase with the number of jobs, which makes it very effective to solve large instances to optimality, especially when multiple jobs have equal size and processing time. We compare our model to other models from the literature, showing its clear superiority on benchmark instances and proving optimality of random instances with up to 100 million jobs.
\end{abstract}

Keywords: scheduling, batch processing machine, makespan, arc-flow, symmetry

\section{Introduction}

This paper addresses Batch Processing Machines (BPM) scheduling, which has been extensively explored in the literature, motivated by applications in industries and by the challenging solution of real-world problems. The main goal in BPM scheduling problems is to group jobs into batches and process them simultaneously on a machine, to facilitate the tasks and to reduce the time spent in handling the material. There are many variations of problems involving BPM with applications in industry and manufacturing, such as the schedule of shuttle buses in the transportation system, the schedule in tire production plants ([43], [30]), and applications in sterilization services in hospitals ([31]). The version of the BPM considered in this work is particularly suitable to applications in the semiconductor industry ([38]) and parallel computing ([1]). Particularly, the first application arises in reliability tests in the semiconductor industry, in operations called "burn-in" (see [38]). The burn-in operation is used to test electronic circuits and consists of assigning them to industrial ovens, subjecting them to thermal stress for a long period of time. The test of each circuit is considered here as a job and requires a minimum time inside the oven, which is the processing machine. The jobs cannot be processed directly on the machine; they need to be placed on a tray, respecting its capacity. Each group of jobs assigned to a tray is considered a batch. The minimum time of the circuit inside the oven is set a priori, based on the supplier requirements. It is assumed that it

\footnotetext{
*Corresponding author.
}

Address: 1 rue Honoré d'Estienne d'Orves, 91120 Palaiseau, France. Email: rst@lix.polytechnique.fr

This is an accepted manuscript version.

DOI: $10.1016 /$ j.cor.2021.105394

(C) 2021. This manuscript version is made available under the CC BY-NC-ND 4.0 license.

(cc) BY-NC-ND 
is possible to keep the circuit in the oven longer than necessary, with no ill effect, but the circuit cannot be removed from the oven before its required processing time is fulfilled. Therefore, the processing time of a batch is determined by the longest processing time among all jobs assigned to it. The burn-in tests are a bottleneck in final testing operations, and the efficient scheduling of these operations aims at maximizing productivity and reducing flow time in the stocks, which are major concerns for management. In [19], [34], and [5], the complexity of burn-in tests on electronic circuits and liquid crystal displays is discussed, reinforcing the importance of efficient scheduling for them.

Compared to the history of semiconductor manufacturing, the interest of researchers in BPM is recent. A review of scheduling models considering batch-processing machines is presented in [32], where the authors point out that the research effort in designing algorithms for these problems is worthwhile. A survey on BPM problems is presented in [24], considering publications between 1986 and 2004 (part of 2004 only). A more recent survey is published in [27].

The BPM problem specifically addressed in this paper is the single batch-processing machine scheduling problem denoted by $1\left|s_{j}, B\right| C_{\max }$, where jobs have non-identical sizes and processing times, and the objective is to minimize the makespan. The problem was reported for the first time in [38], where its NP-hard complexity is proven and a heuristic approach to solve it is proposed. Heuristics are also proposed for this problem in [13] and [12], where instances with up to 100 jobs are considered. Two approximation algorithms are presented in [42], with approximation ratios of $3 / 2$ and $7 / 4$ of the optimal solution value in the worst case. In [25], the simulated annealing metaheuristic was applied to $1\left|s_{j}, B\right| C_{\max }$, and a Mixed Integer Linear Programming (MILP) formulation was presented for the problem. [25] also propose configurations for test instances that were widely used in later works. Computational results are shown for instances with up to 100 jobs, comparing the heuristic solutions to the solutions obtained with the MILP formulation. Other meta-heuristics are also applied to problem $1\left|s_{j}, B\right| C_{\max }$ in the literature, namely, genetic algorithm ([8] and [15]), tabu search ([26]), and GRASP ([7]). These four papers consider instances with up to 100 jobs as well. In [4], a heuristic based on a special case of the clustering problem is proposed, and test instances with up to 500 jobs are considered. In [20], two heuristics are proposed based on a decomposition of the original problem, where relaxations of the problem are solved. Instances with up to 100 jobs are considered in this work. The ant colony optimization algorithm is applied to this problem in [14]. The bee colony meta-heuristic is also applied to the problem in [1], where results for instances with up to 200 jobs are considered. In [21], the authors propose enumeration schemes to improve the heuristics proposed in [13]. The schemes are able to find good solutions for instances of up to 500 jobs in less than $0.25 \mathrm{~s}$. The works mentioned above do not share a common set of instances, which makes a direct comparison regarding the quality of the solutions difficult to perform. Concerning metaheuristics, the colony algorithm proposed by [14] shows superior results compared to the clustering algorithm, the genetic algorithm and simulated annealing. Considering heuristics, [20] and [21] present very fast methods, which are good alternatives for finding solutions of large-sized problems in less than $1 \mathrm{~s}$.

Most publications addressing problem $1\left|s_{j}, B\right| C_{\max }$ apply heuristic and meta-heuristic approaches. Nevertheless, exact methods are also applied in the literature, as for example in [11], [33], [37], [35], and [29]. In [11], a branch-and-bound procedure is developed considering dominance properties for a general enumeration. This procedure is also used as a heuristic for large instances. In [33], a formulation for $1\left|s_{j}, B\right| C_{\max }$ is presented using a partition problem in the context of Dantzig-Wolfe decomposition. A branch-and-price algorithm is applied to solve the problem to optimality. Instances with up to 500 jobs are considered in the computational experiments. In [37] and [35], the authors consider the exact solution of the problem, improving the formulation presented in [25]. Sets of symmetric solutions for the formulation are identified. Symmetry breaking constraints are then proposed, taking into account specific properties of the problems and their optimal solutions to propose new stronger formulations and avoid undesirable symmetric solutions in their feasible sets. [29] proposes a column-and-cut generation (CCG) algorithm for the instances that could not be solved to optimality with the model proposed by [37]. The method consists of running the model proposed by [37] for 300 seconds to obtain an upper bound or, if possible, an optimal solution, and then running the GCC method to improve the bounds. This work also proposes an exact algorithm for the version of the problem that considers parallel machines.

(C) 2021. This manuscript version is made available under the CC BY-NC-ND 4.0 license. 
In this paper, we aim at solving problem $1\left|s_{j}, B\right| C_{\max }$ to optimality as well, but considering an arc-flow optimization approach. Arc-flow formulations from the literature, for classical combinatorial optimization problems, make use of a pseudopolynomial number of variables and constraints. For example, see [39], where the author proposes a branch-and-price algorithm for an arc-flow based formulation for a cutting-stock problem. Later, the methodology was extended for the bin-packing problem in [40]. An alternative arc-flow formulation for the cutting-stock problem is proposed in [2] and [3], using graph compression technique. These formulations were recently tested and compared in [10] against several other models and problem-specific algorithms on one-dimensional bin packing and cutting stock problems. The results show that the arc-flow based formulations outperform all other models. [22] extended the model proposed by Valério de Carvalho for the two-dimensional cutting stock problem. The authors tested the resulting model on a set of real instances derived from the furniture industry, and it proved to be more efficient than previous methods. In [23], the arc-flow model and the one-cut model are compared for the one-dimensional cutting-stock problem, and reduction techniques for both approaches are presented. [6] introduce new sets of patterns for cutting and packing problems as, for example, arc-flow formulations. These patterns can also be applied to several other problems. Particularly considering scheduling problems, we are aware of some recent papers that apply an arc-flow approach. In [28], the makespan minimization problem on identical parallel machines, $P \| C_{m a x}$, is considered. In [17], problem $P \| \sum w_{j} C_{j}$ is considered, where the goal is to schedule a set of jobs on a set of identical parallel machines, while minimizing the total weighted completion time. Different versions of the problem are presented in [16], where release times $P\left|r_{j}\right| \sum w_{j} C_{j}$ are considered, and in [18], where family dependent setup times $P\left|s_{i}\right| \sum w_{j} C_{j}$ are considered. A broader discussion of the methods and applications of the arc-flow models can be found in [9]. In [9], theoretical foundations for pseudo-polynomial arc-flow formulations are presented. To our knowledge, our work is the first to consider arc-flow formulations for scheduling problems on batch processing machines.

Motivated by the good results obtained with arc-flow approaches for combinatorial problems, we present in this paper a novel arc-flow based formulation for problem $1\left|s_{j}, B\right| C_{\max }$, representing it as a problem of determining flows in graphs. A special graph $G$ and arc-flow structures corresponding to the distinct processing times of the jobs are introduced to form the core of our formulation. With the approach used in the modeling, we are able to propose a formulation where neither the number of variables nor the number of constraints increases with the number of jobs. On the other hand, they increase with the machine capacity, i.e, with the batch size and with the number of distinct sizes and processing times among the jobs. In this way, we see our model as an alternative option to other models from the literature, where the number of the variables depends on the number of jobs, but does not depend on these other features. Our model is particularly effective for solving large instances to optimality when the machine capacity is not too large in comparison to the sizes of the jobs, and when we have multiple jobs with equal sizes and processing times. Another important feature of the formulation is that it does not contain some of the symmetries contained in the formulations presented in [33] and [37]. We present numerical experiments where very large instances of the problem, with a number of jobs never considered in the literature, are solved to optimality in short times. In fact, the solution time for these large instances are smaller than the solution time of much smaller instances, when using other formulations from the literature. After the development of this work, we could also successfully extend the idea of the arc-flow model to the case of parallel machines. Computational results for this extension can be found in [36].

We organize the paper as follows: In Section 2, we introduce problem $1\left|s_{j}, B\right| C_{\max }$ and present two formulations from the literature. In Section 3, we present an arc-flow based formulation for the problem. In Section 4, we discuss our numerical experiments comparing the arc-flow formulation to formulations from the literature. In Section 5, we present some concluding remarks and discuss future work.

(C) 2021. This manuscript version is made available under the CC BY-NC-ND 4.0 license. 


\section{Problem definition}

The single batch-processing machine scheduling problem, or problem $1\left|s_{j}, B\right| C_{\max }$, can be formally defined as follows. Given a set $J:=\left\{1, \ldots, n_{J}\right\}$ of jobs, each job $j \in J$ has a processing time $p_{j}$ and a size $s_{j}$. Each job must be assigned to a batch $k \in K:=\left\{1, \ldots, n_{K}\right\}$, respecting the given capacity $B$ of the machine, so that the sum of the sizes of the jobs assigned to a batch cannot exceed $B$. We assume that $s_{j} \leq B$, with $s_{j}, B \in \mathbb{Z}$, for all $j \in J$. The batches must be all processed in a single machine, one at a time, and all the jobs assigned to a single batch are processed simultaneously. The processing time $\tau_{k}$ of each batch $k \in K$ is defined as longest processing time among all jobs assigned to it, i.e., $\tau_{k}:=\max \left\{p_{j}: j\right.$ is assigned to $\left.k\right\}$. Jobs cannot be split between batches. It is also not possible to add or remove jobs from the machine while the batches are being processed. The goal is to schedule the jobs on the batches so that the makespan $\left(C_{\max }\right)$ is minimized, where the makespan is defined as the time required to finish processing the last batch.

The number of batches used in the solution of $1\left|s_{j}, B\right| C_{\max }$ is not fixed and should be optimized. It will depend on the number of jobs, their sizes, and the machine capacity. In this section, we present two formulations from the literature to solve the $1\left|s_{j}, B\right| C_{\max }$, where $n_{K}$ represents an upper bound on the number of batches allowed in a solution. We will notice that this value impacts in the size of these formulations.

In the worst case, each job is assigned to a different batch, and the number of batches used is equal to the number of jobs $n_{J}$. It is important to note that even when it is possible to find a feasible solution for a problem where the number of batches used is $\phi<n_{J}$, we should not limit the maximum number of batches to $\phi$ in the problem's formulation, as we should consider the case in which creating an additional batch in the solution would decrease the makespan. As an example of this situation, we show in Figure 1 two feasible solutions for an instance of $1\left|s_{j}, B\right| C_{\max }$. Although the number of batches used in solution $(a)$ is smaller than in solution $(b)$, its makespan is bigger. Therefore, to assure the correctness of the formulations presented next, it is always considered $n_{K}=n_{J}$.

a)

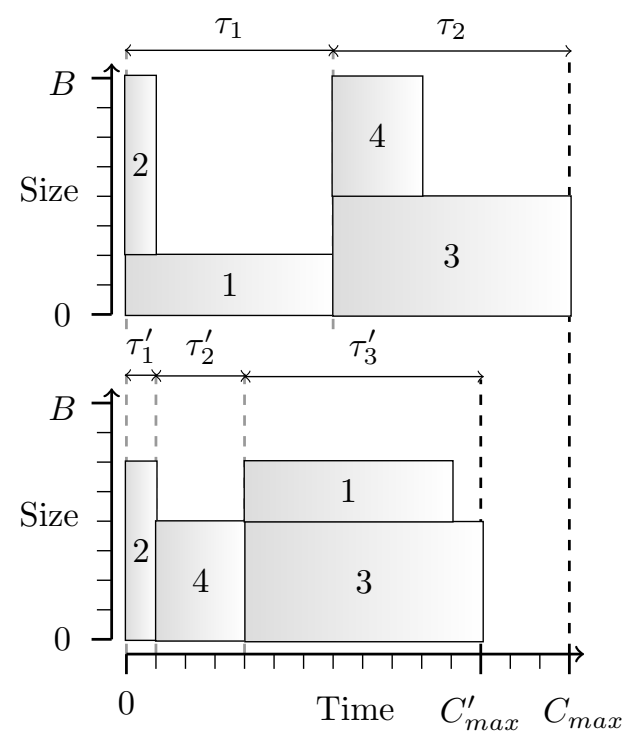

Figure 1: Feasible solutions with (a) 2 batches and (b) 3 batches.

Let us consider the following decision variables for all $j \in J, k \in K$ :

$$
\begin{aligned}
& x_{j k}= \begin{cases}1, & \text { if job } j \text { is assigned to batch } k, \\
0, & \text { otherwise, }\end{cases} \\
& y_{k}= \begin{cases}1, & \text { if batch } k \text { is used, } \\
0, & \text { otherwise, }\end{cases} \\
& \tau_{k} \quad \text { : processing time of batch } k \text {. }
\end{aligned}
$$

(C) 2021. This manuscript version is made available under the CC BY-NC-ND 4.0 license. 
In [25] the following MILP formulation (1)-(7) is proposed for problem $1\left|s_{j}, B\right| C_{\max }$. Other very similar formulations and sometimes this exactly same one, are used in other papers as a comparative basis in computational experiments.

$$
\begin{aligned}
& \left(\mathrm{MILP}_{1}\right) \min \sum_{k \in K} \tau_{k}, \\
& \sum_{k \in K} x_{j k}=1, \\
& \sum_{j \in J} s_{j} x_{j k} \leq B y_{k}, \\
& \forall j \in J, \\
& \tau_{k} \geq p_{j} x_{j k}, \\
& x_{j k} \leq y_{k} \text {, } \\
& y_{k} \in\{0,1\} \text {, } \\
& x_{j k} \in\{0,1\} \text {, } \\
& \forall k \in K, \\
& \forall j \in J, \forall k \in K, \\
& \forall j \in J, \forall k \in K \text {, } \\
& \forall k \in K, \\
& \forall j \in J, \forall k \in K \text {. }
\end{aligned}
$$

The objective function (1) minimizes the makespan, given by the sum of the processing times of all batches. Constraints (2) determine that each job is assigned to a single batch. Constraints (3) determine that the machine capacity is respected. Constraints (4) determine the processing times of the batches. Note that constraints (5) are redundant because of (3), but are added to strengthen the linear relaxation of the formulation. Constraints (6) and (7) define the domains of the variables.

In [37], the authors address the symmetry in formulation $\left(\mathrm{MILP}_{1}\right)$. They present a new formulation for the problem, where symmetric solutions are eliminated from the feasible set of $\left(\mathrm{MILP}_{1}\right)$, with the following approach. Firstly, the indexes of the jobs are defined by ordering them by nondecreasing order of their processing times, i.e., $p_{1} \leq p_{2} \leq \ldots \leq p_{n_{J}}$. Secondly, it is determined that batch $k$ can only be used if job $k$ is assigned to it, for all $k \in K$. Thirdly, it is determined that job $j$ can only be assigned to batch $k$ if $j \leq k$. Considering the above, the following formulation for $1\left|s_{j}, B\right| C_{\max }$ is proposed in [37]:

$$
\begin{gathered}
\left(\mathrm{MILP}_{1}^{+}\right) \min \sum_{k \in K} p_{k} x_{k k}, \\
\sum_{k \in K: k \geq j} x_{j k}=1, \\
\sum_{j \in J: j \leq k} s_{j} x_{j k} \leq B x_{k k}, \\
x_{j k} \leq x_{k k}, \\
x_{j k} \in\{0,1\},
\end{gathered}
$$

The objective function (8) minimizes the makespan, given by the sum of the processing times of the batches used. Constraints (9) determine that each job $j$ is assigned to a single batch $k$, such that $k \geq j$. Constraints (10) determine that the machine capacity is respected. They also ensure that each batch $k$ is used if and only if job $k$ is assigned to it. Constraints (11) are redundant, since they have already been imposed by (10), but they are still included to strengthen the linear relaxation of the model.

It is important to notice that both formulations MILP and MILP ${ }_{1}^{+}$allow intermediate empty batches when representing the solutions. Although this leads to symmetries in the feasible set of MILP, where a solution can be represented with different indices attributed to the same batches, this symmetry is eliminated in MILP $_{1}^{+}$as discussed in [37].

We note that there are similarities between problem $1\left|s_{j}, B\right| C_{\max }$ and the classic bin-packing and cutting-stock combinatorial optimization problems. [38] proves that the bin-packing problem is a special case of $1\left|s_{j}, B\right| C_{\max }$, in which the processing times $p_{j}$, for all jobs, are equal to one. Therefore, any formulation for $1\left|s_{j}, B\right| C_{\max }$ can also model the bin-packing problem. The opposite, on the other hand, is not valid, because formulations for bin packing cannot distinguish items with

(C) 2021. This manuscript version is made available under the CC BY-NC-ND 4.0 license. 
different processing times, neither calculate the makespan. Moreover, the cutting-stock problem can be seen as a generalization of the bin-packing problem, with unit demands, as shown in [41] and [10]. However, it is not a generalization of problem $1\left|s_{j}, B\right| C_{\max }$. As the cost of each pattern in the cutting-stock problem is originally set to one, the different processing times of the batches in the $1\left|s_{j}, B\right| C_{\max }$ problem, cannot be represented in the cutting-stock formulation.

\section{An arc-flow based optimization approach}

In this section we propose an arc-flow based optimization approach to problem $1\left|s_{j}, B\right| C_{\max }$, formulating it as a problem of determining flows in graphs. The arc-flow approach was also applied to formulate other problems, such as the cutting-stock problem, in [39], and the bin-packing problem, in [40]. It is important to note, however, that the formulations for these problems cannot be trivially applied to $1\left|s_{j}, B\right| C_{\max }$, as mentioned in the previous section. Some specific aspects of this last problem have to be exploited and considered in the new formulation that we propose in the following.

We initially define a directed graph $G:=(V, A)$, in which each (integer) physical space of the batch with capacity $B$ is represented by a node, so

$$
V:=\{0,1, \ldots, B\} .
$$

The set of directed $\operatorname{arcs} A$ is divided into three subsets: the set of job arcs $A^{J}$, the set of loss $\operatorname{arcs} A^{L}$, and the set with a feedback arc $A^{F}$. Therefore, $A=A^{J} \cup A^{L} \cup A^{F}$.

Each arc $(i, j)$ of the subset $A^{J}$ represents the existence of at least one job $k$ of size $s_{k}$, such that $s_{k}=j-i$. More specifically, the subset $A^{J}$ is defined as:

$$
A^{J}:=\left\{(i, j): \exists k \in J \mid s_{k}=j-i, i, j \in V, i<j\right\} .
$$

As it will become clear with the presentation of our arc-flow formulation in Subsection 3.4, to compose valid paths and represent all possible solutions for the problem, it is necessary to include the loss arcs in $G$, which represent empty spaces in the batches. More specifically, the subset of arcs $A^{L}$ is defined as:

$$
A^{L}:=\{(i, B): i \in V \backslash\{0, B\}\}
$$

Note that the loss arcs are all incident to the last node of the batch. This representation allocates all the empty spaces at the end of the batch and eliminates some symmetric solutions where empty spaces appear between two allocated jobs.

Finally, the feedback arc is used to connect the last node to the first one, defined as:

$$
A^{F}:=\{(B, 0)\}
$$

Figure 2 shows the graph $G$ for an instance of $1\left|s_{j}, B\right| C_{\max }$, with 5 jobs. The machine capacity and the sizes of the jobs are presented in Table 1.

\begin{tabular}{c|ccccc}
\hline Machine capacity & \multicolumn{5}{|c}{ Job size } \\
$B$ & $s_{1}$ & $s_{2}$ & $s_{3}$ & $s_{4}$ & $s_{5}$ \\
\hline 5 & 3 & 2 & 2 & 1 & 1 \\
\hline
\end{tabular}

Table 1: Example of problem $1\left|s_{j}, B\right| C_{\max }$

In our modeling approach, each unit flow in graph $G$, going from node 0 to node $B$, represents the configuration of a batch in the solution of the problem. Moreover, a unit flow from node $B$ to node 0 , going through the feedback arc, represents a solution using a single batch, and flows with more than one unit on the feedback arc, represent the use of several batches.

As all flows are non-negative and $G$ is acyclic once the feedback arc is excluded, it is possible to decompose the multiple flow on the feedback arc into several unit flows, each corresponding to

(C) 2021. This manuscript version is made available under the CC BY-NC-ND 4.0 license. 


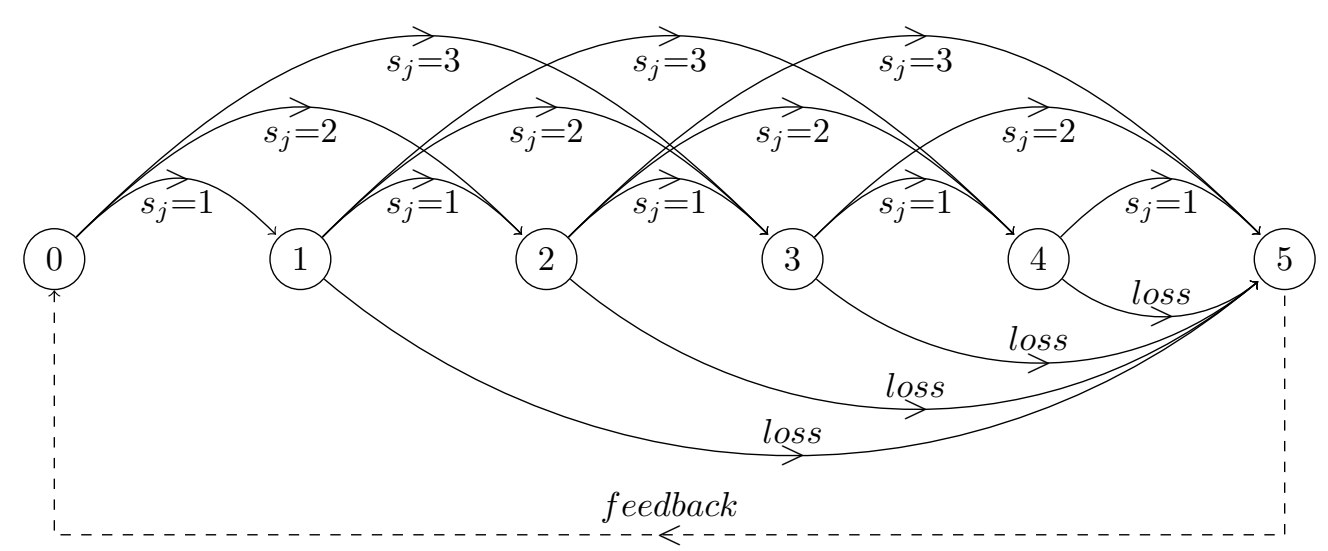

Figure 2: Graph $G$ representing the arc-flow structure used to model the instance of $1\left|s_{j}, B\right| C_{\max }$ in Table 1.

an oriented path from node 0 to node $B$. Each unit flow represents the configuration of a different batch and the flow on the feedback arc indicates the number of batches used in the solution.

Figure 3(a) depicts a particular solution for the instance of problem $1\left|s_{j}, B\right| C_{\max }$ described in Table 1, which is represented by the graph $G$ in Figure 2. The multiple-flow graph of two units is decomposed into two other graphs in Figures 3(b) and 3(c), each with a unit flow. They represent two batches 1 and 2, such that jobs $\{1,4,5\}$ are assigned to batch 1 (Figure 3(b)) and jobs $\{2,3\}$ are assigned to batch 2 (Figure 3(c)). Arcs with null value are not shown. We note this decomposition of the multiple-flow arc is not unique. The loss arc and the job arc corresponding to the fifth job could be exchanged between the graphs in Figures 3(b) and 3(c).

The graph $G$ defined above, is then replicated for each distinct processing time of the problem in our modeling approach. Each replicated graph will be referred to, as an arc-flow structure for our problem. We define $P:=\left\{P_{1}, \ldots, P_{\delta}\right\}$ as the set with all the distinct processing times among all jobs, and $T:=\{1, \ldots, \delta\}$ as the set of indexes corresponding to the arc-flow structures in the problem formulation. We consider $P_{1}<P_{2}<\ldots<P_{\delta}$. The arc-flow structure $t \in T$, will have the corresponding processing time fixed at $P_{t}$, meaning that all batches represented in the arc-flow structure $t$ will be considered in our formulation to have processing time $P_{t}$. Only jobs $j$ with processing time $p_{j} \leq P_{t}$ will be allowed to be assigned to this arc-flow structure. Moreover, the minimization of the makespan in the objective function will assure that all batches represented in the arc-flow structure $t$ in the optimal solution of the problem, have at least one job with processing time $P_{t}$ assigned to it. The number of batches corresponding to each arc-flow structure is indicated by the value of the flow in the feedback arc. As each arc-flow structure can represent as many batches as needed, it is possible to represent all solutions of problem $1\left|s_{j}, B\right| C_{\max }$ with our arc-flow structures. Finally, we note that, in the optimal solution of the problem, the maximum number of batches represented in the arc-flow structure $t$ is given by the number of jobs with processing time $P_{t}$. This bound will only be attained when each job with processing time $P_{t}$ is assigned to a different batch.

In Figure 4, we illustrate a feasible solution for an instance of problem $1\left|s_{j}, B\right| C_{\max }$ with fifteen jobs. The processing times for the jobs are either 3, 4, or 5. They are grouped into six batches, two of them per processing time. We want to emphasize here, that the number of the arc-flow structures used in our formulation is given by the number of distinct processing times on the instance. Therefore, for this example, only three structures are used to represent the feasible solutions. Particularly, the solution in Figure 4 is represented by the three structures in Figure 5, one for each distinct processing time.

The graph $G$ and the arc-flow structures introduced above form together the core of the arc-flow formulation for problem $1\left|s_{j}, B\right| C_{\max }$. It is important then to analyze what are the parameters of

(C) 2021. This manuscript version is made available under the CC BY-NC-ND 4.0 license. 


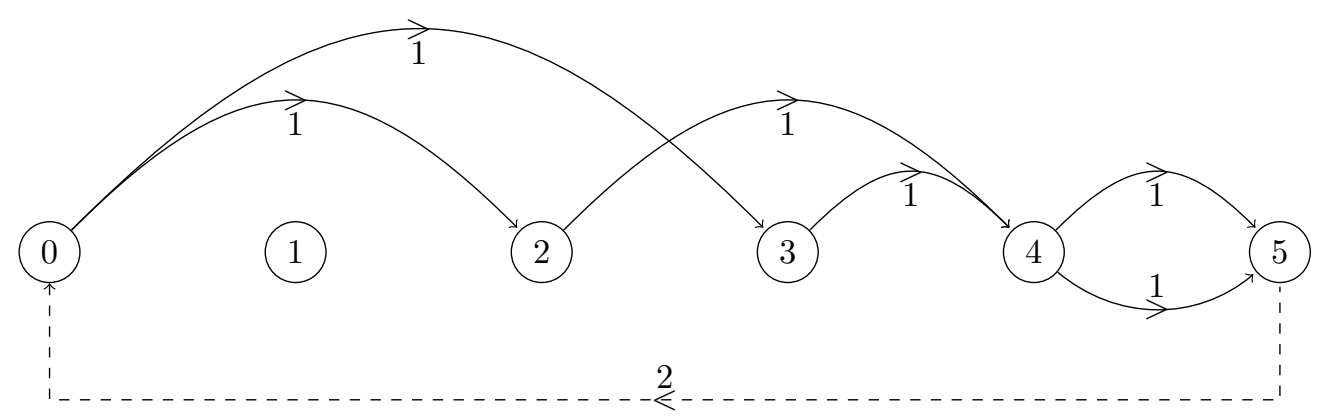

(a)

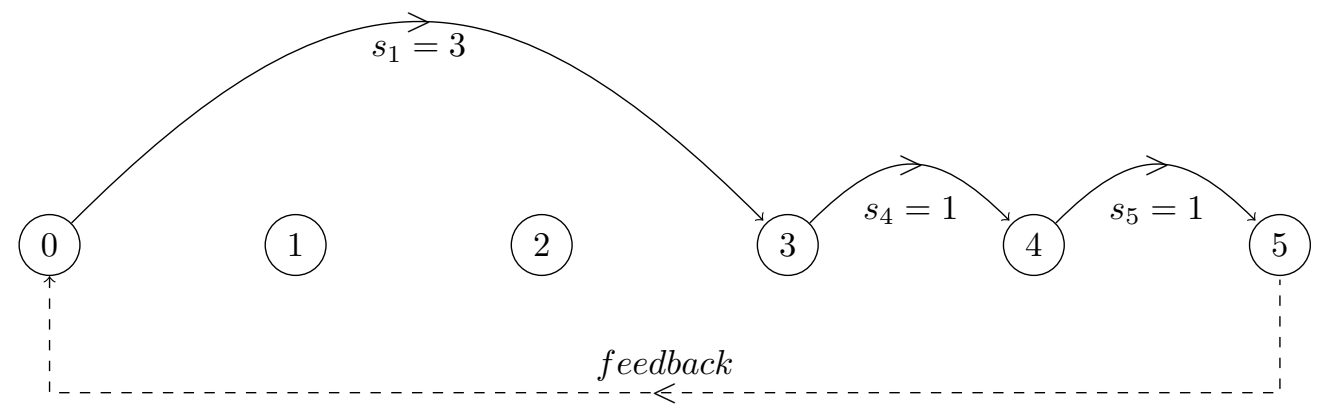

(b)

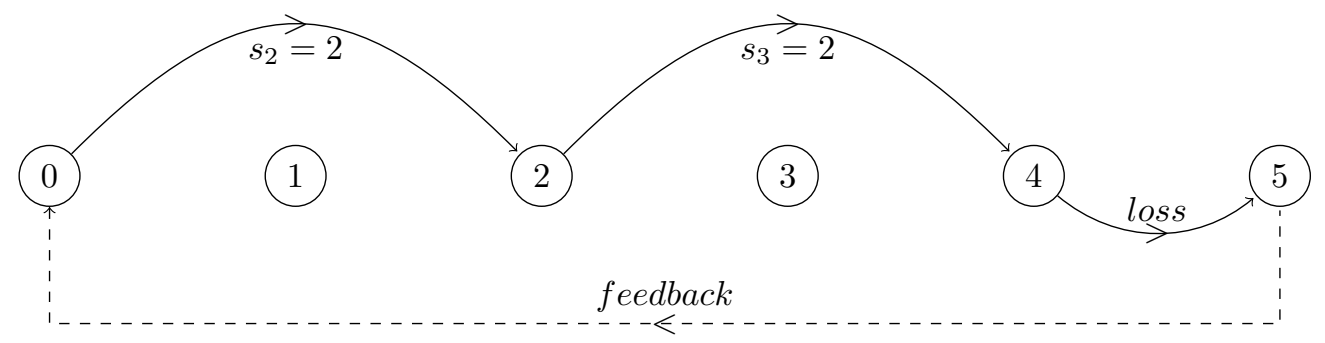

(c)

Figure 3: (a) Feasible solution for problem represented in Figure 2, with multi-flow decomposed into (b) and (c).

the problem that interfere in the size and density of $G$ and also in the number of arc-flow structures that are needed to represent the solution. These are the parameters that will interfere in the size of our problem formulation. We emphasize them in the following remark.

Proposition 1. Consider problem $1\left|s_{j}, B\right| C_{\max }$, with machine capacity $B \in \mathbb{Z}$. There are a set of jobs to be assigned to batches, with $\delta$ distinct processing times $\left\{P_{1}, P_{2}, \ldots, P_{\delta}\right\}$ and $\theta$ distinct sizes $\left\{S_{1}, S_{2}, \ldots, S_{\theta}\right\}$. Consider the graph $G=(V, A)$ and the arc-flow structures defined as above to represent a feasible solution to $1\left|s_{j}, B\right| C_{\max }$. We have $|V|=B+1,|A|=\theta+(\theta+1) B-\sum_{i=1}^{\theta} S_{i}$, and the number of arc-flow structures needed to represent all feasible solutions equals to $\delta$.

Proof. The deduction of $|V|$ and the number of arc-flow structures is straightforward. To deduce the number of arcs $|A|$, we should note that it is the sum of the number of arcs in the three subsets $A^{J}$, $A^{L}$ and $A^{F}$. Considering (14), we show in Table (2), the number of arcs that are incident to each

(C) 2021. This manuscript version is made available under the CC BY-NC-ND 4.0 license. 


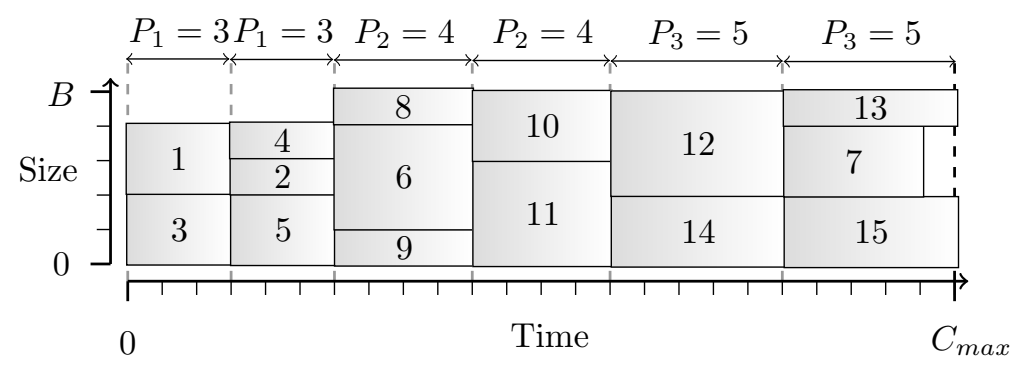

Figure 4: Solution with $C_{\max }=24$ and six batches.

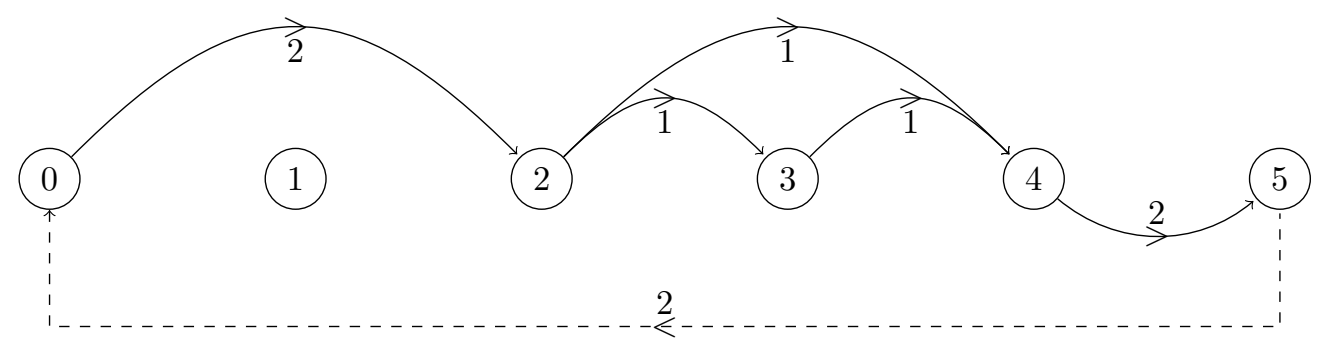

(a) Representation of batches with processing time $P_{1}=3$

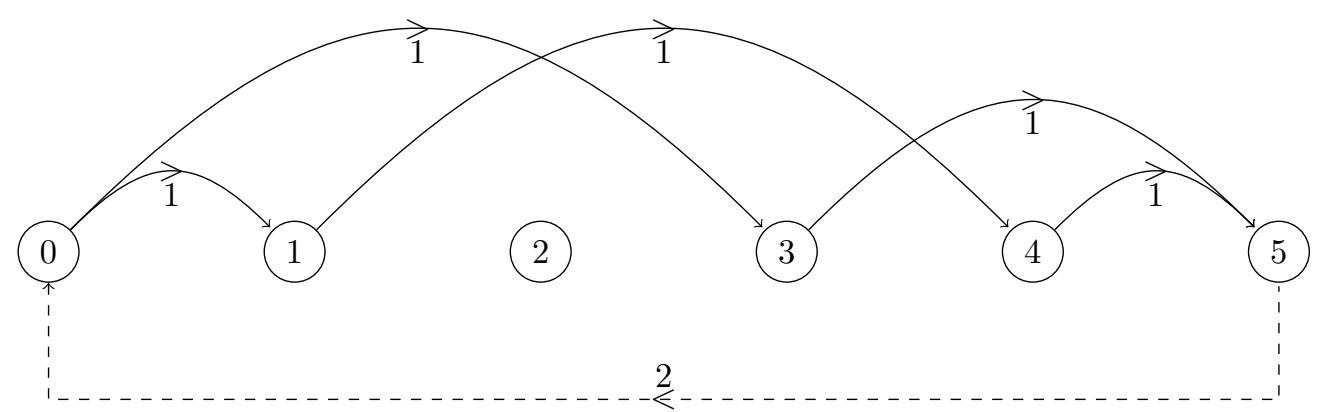

(b) Representing of batches with processing time $P_{2}=4$

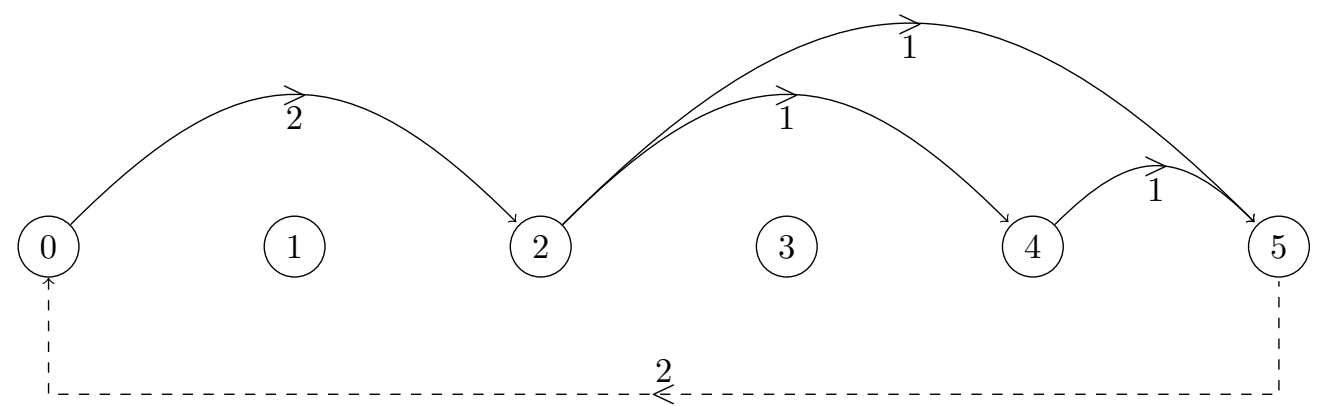

(c) Representation of batches with processing time $P_{3}=5$

Figure 5: Arc-flow structures representing the solution in Figure 4. 
node $j$ in $A^{J}$, when $j$ belongs to each of the intervals presented. The sum of the incident arcs over all nodes $j$ in each interval is also shown. Adding, then, the number of arcs over all the intervals,

\begin{tabular}{lcc}
\hline node & $\begin{array}{c}\text { \# arcs incident } \\
\text { to each node } j\end{array}$ & $\begin{array}{c}\text { \# arcs incident } \\
\text { to all nodes } j\end{array}$ \\
\hline$j \in\left[0, S_{1}-1\right]$ & 0 & 0 \\
$j \in\left[S_{1}, S_{2}-1\right]$ & 1 & $1 \times\left(S_{2}-S_{1}\right)$ \\
$j \in\left[S_{2}, S_{3}-1\right]$ & 2 & $2 \times\left(S_{3}-S_{2}\right)$ \\
\multicolumn{1}{c}{$\vdots$} & $\vdots$ & $\vdots$ \\
$j \in\left[S_{\theta-1}, S_{\theta}-1\right]$ & $\theta-1$ & $(\theta-1) \times\left(S_{\theta}-S_{\theta-1}\right)$ \\
$j \in\left[S_{\theta}, B\right]$ & $\theta$ & $\theta \times\left(B-S_{\theta}+1\right)$ \\
\hline
\end{tabular}

Table 2: Arcs on $A^{J}$

we obtain $\left|A^{J}\right|=\theta(B+1)-\sum_{i=1}^{\theta} S_{i}$. Moreover, from (15), we have $\left|A^{L}\right|=B-1$, and from (16), we have $\left|A^{F}\right|=1$, which completes the proof.

We observe from Proposition 1 that the size of the proposed arc-flow formulation for problem $1\left|s_{j}, B\right| C_{\max }$ (that we present in Subsection 3.4), increases with the number of distinct sizes and processing times among the jobs, but does not increase with the number of jobs. In fact, the number of decision variables on our proposed formulation depends on the number of arcs on the arc-flow structures used to model the problem. This makes the formulation particularly suitable for large instances where there are multiple jobs with similar characteristics, specifically with equal size and processing time.

\subsection{Mapping arc's flows into job assignments and breaking symmetries}

The solution represented by the arc-flow structure described above, does not specify exactly which are the jobs assigned to each batch, but only the number of jobs with each size that are assigned to it. For example, if we decompose the two-units multi-flow structure in Figure 5(a) into two unit-flow structures, we can easily see that it represents two batches with processing time $P_{1}=3$. One of them has two jobs of size $s=2$ and the other has two jobs of size $s=1$ and one job of size $s=2$. As shown in Figure 4, the instance considered has three jobs of size $s=2$ and processing time $P_{1}=3$, jobs 1,3 , and 5 . Therefore, each one of them could be assigned to the latter batch, generating three equivalent solutions.

Moreover, the arc-flow solution determines the number of batches with each processing time, but defines no processing ordering for them. As in problem $1\left|s_{j}, B\right| C_{\max }$, release times are not considered for the jobs, any permutation in the ordering of the batches would also lead to equivalent solutions.

Frequently, mathematical programming formulations for combinatorial optimization problems, make distinction between equivalent solutions by indexing them in different ways. These are called symmetric solutions, and their existence in the feasible set of the problems tends to worsen the performance of branch-and-bound algorithms for wasting effort evaluating equivalent solution unnecessarily. In [37], the authors point out that this is the case of formulation (MILP 1 ), and show that the symmetry generated by exchanging the indexes assigned to the batches in the solution of $\left(\mathrm{MILP}_{1}\right)$ is broken in $\left(\mathrm{MILP}_{1}^{+}\right)$. We note, however, that the symmetry generated by exchanging the indexes assigned to jobs with the same sizes and processing times is still not broken in $\left(\mathrm{MILP}_{1}^{+}\right)$.

The arc-flow formulation has neither of these symmetries in its feasible set, and with a simple mapping, we can determine a feasible assignment of jobs to batches, according to the arc-flow optimal solution. The mapping of arcs into jobs is easily performed in polynomial time, since each job arc $(i, j)$ in the arc-flow structure corresponding to processing time $P_{t}$, can be mapped into any job of size $j-i$ and processing time not greater than $P_{t}$. In order to guarantee that a feasible solution is obtained, we should only map the arcs into jobs starting from the structures with the smallest processing time and continue in increasing order of processing time. Concerning structures with the same processing times, the ordering for the mapping among them is not relevant.

(C) 2021. This manuscript version is made available under the CC BY-NC-ND 4.0 license. 
Finally, we should observe that, although we have eliminated symmetries contained in the feasible sets of $\left(\mathrm{MILP}_{1}\right)$ and $\left(\mathrm{MILP}_{1}^{+}\right)$with the arc-flow formulation, it still contains symmetric solutions in its feasible set, related to the "positions" occupied by the jobs in the batches, as determined by the definition of the variables of the formulation presented in the following.

\subsection{Arc reduction}

Some rules can be set to decrease the number of arcs in graph $G$ and the number of variables in our arc-flow formulation. The set $A^{L}$ of loss arcs defined in (15), represents empty spaces that occur at the end of the batch. We first note that our modeling does not represent empty spaces between jobs or at the beginning of the batch, which avoids symmetric solutions on the feasible set of the problem. Because of that, two rules can be created to eliminate arcs that will never be used in the solution of the problem: Rule 1 eliminates job arcs that cannot be used in a valid flow starting at node 0 , and Rule 2 eliminates loss arcs incident to nodes that are not incident to any job arc.

Rule 1. Only job $\operatorname{arcs}(i, j) \in A^{J}$ that belong to at least one continuous flow starting at node 0 can have a positive flow in the solution of the problem, and therefore, all the others may be eliminated from graph $G$. The job arcs that belong to at least one continuous flow can be selected by the following steps:

1. arc $(0, j)$ is selected, for all $j$, such that $(0, j) \in A^{J}$;

2. $\operatorname{arc}(i, j) \in A^{J}$ is selected, if an arc $(k, i)$ has been previously selected for some node $k$;

3. repeat step (2) until no arc is selected.

Rule 2. Only loss arcs $(i, B) \in A^{L}$ that are incident to a node $i$, which is incident to a remaining job arc after the application of Rule 1, can have a positive flow in the solution of the problem, and therefore, all the others may be eliminated from graph $G$.

These rules are similar to those discussed in [40, Criterion 1-2] and widely applied in arc-flow formulations in the literature. One difference between our formulation and the formulation in [40], however, is that in [40] the unit loss arcs connect nodes $i$ and $i+1$, while in our formulation they connect nodes $i$ and $B$.

Figure 6 illustrates the application of Rules 1-2, where 2 job arcs and 1 loss arc are removed from graph $G$.

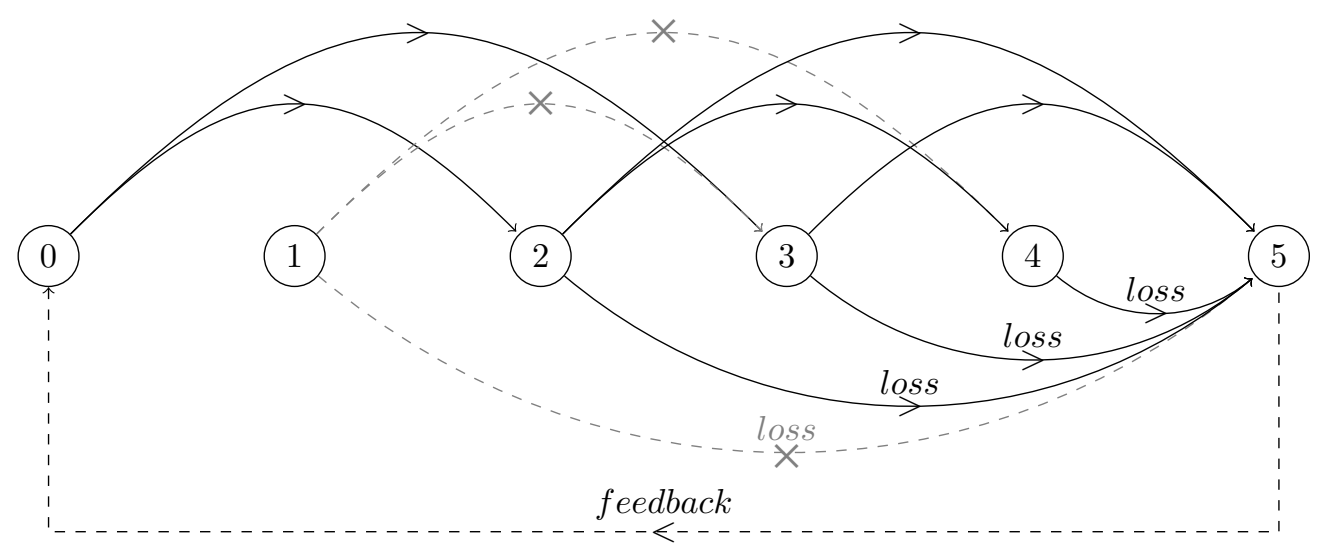

Figure 6: Example of arc reduction.

(C) 2021. This manuscript version is made available under the CC BY-NC-ND 4.0 license. 


\subsection{Defining bounds for the variables in the formulation}

In the following, we define bounds on the values of the flows in each arc of the arc-flow structures, and therefore bounds for the variables in the proposed model.

Rule 3. The flow on each arc $(i, j) \in A$ that belongs to the arc-flow structure corresponding to processing time $p$ cannot be greater than the number of jobs with processing time $p$. This upper bound is more specifically given by

$$
\sum_{k \in J: p_{k}=p} 1 .
$$

Rule 4. The flow on each job arc $(i, j) \in A^{J}$ that belongs to the arc-flow structure corresponding to processing time $p$ cannot be greater than the number of jobs of size $j-i$ and processing time not greater than $p$. This upper bound is more specifically given by

$$
\sum_{\substack{k \in J: s_{k}=j-i, p_{k} \leq p}} 1 .
$$

\subsection{Arc-flow formulation}

In this section, we formulate problem $1\left|s_{j}, B\right| C_{\max }$ as the problem of determining the flows from node 0 to node $B$ on the arc-flow structures associated to the distinct processing times of the jobs. The objective is to minimize the sum of the flows on the structures, weighted by their associated processing times. We emphasize that the flow on the structure associated to the processing time $P_{t}$ indicates the number of batches with processing time $P_{t}$ in the solution, therefore, the objective function of our model represents the makespan of the batch-processing machine.

Besides the set of variables and constraints that determine the flows on the arcs of the structures and ensure the flow conservation on the nodes, a set of variables and constraints is also required to ensure that all jobs are assigned to an arc-flow structure (or equivalently, to a batch), and that each structure considers the correct number of jobs of each size that are available to be assigned to it.

We recall that our approach requires the arc-flow structures to be sorted by increasing processing time, i.e., the structure $t$ is associated to the processing time $P_{t}$, and $P_{1}<P_{2}<\ldots<P_{\delta}$, where $\delta$ is the number of distinct processing times among all jobs. Let us then suppose that the arc-flow structure $t$ has $\beta$ jobs of size $S_{\ell}$ still available to be assigned to it, where, considering $\theta$ to be the number of distinct sizes among all jobs, we have $\ell \in\{1, \ldots, \theta\}$. These are all the jobs of size $S_{\ell}$ and processing time not greater than $P_{t}$, that have not already been assigned to an arc-flow structure with corresponding processing time smaller than $P_{t}$. A variable $z_{\ell, t}$ is defined in our model to represent how many of these $\beta$ jobs of size $S_{\ell}$ were not assigned to the arc-flow structure $t$. As $\beta$ is the total number of jobs available and assuming that $\alpha$ is the number of jobs that were assigned to structure $t$, the variable $z_{\ell, t}$ assumes value $\beta-\alpha$. This variable will "offer" the remaining $\beta-\alpha$ jobs of size $S_{\ell}$ to the next arc-flow structure, $t+1$, corresponding to next processing time of the problem, greater than $P_{t}$. In the end, all jobs should be assigned to a structure, and, therefore, we should have $z_{\ell, \delta}=0$.

Our arc-flow formulation is presented next.

\section{Sets and parameters}

$B$ : machine capacity.

$\delta$ : number of distinct processing times among all jobs.

$P:=\left\{P_{1}, \ldots, P_{\delta}\right\}:$ set of distinct processing times of jobs, where $P_{1}<P_{2}<\ldots<P_{\delta}$.

$T:=\{1, \ldots, \delta\}:$ set of indexes of the arc-flow structures.

$\theta$ : number of distinct sizes among all jobs.

$S:=\left\{S_{1}, \ldots, S_{\theta}\right\}$ : set of distinct sizes of jobs.

(C) 2021. This manuscript version is made available under the CC BY-NC-ND 4.0 license. 
$N T_{\ell, t}:=\sum_{\substack{j \in J: s_{j}=S_{\ell}, p_{j}=P_{t}}} 1:$ number of jobs of size $S_{\ell}$ and processing time $P_{t}$, for $\ell=1, \ldots, \theta, t \in T$.

$N T_{\ell, t}^{+}:=\sum_{\substack{j \in J: s_{j}=S_{\ell}, p_{j} \leq P_{t}}} 1:$ number of jobs of size $S_{\ell}$ and processing time $\leq P_{t}$, for $\ell=1, \ldots, \theta, t \in T$.

$N J_{t}:=\sum_{\substack{j \in J \\ p_{j}=P_{t}}} 1:$ number of jobs with processing time $P_{t}$, for $t \in T$.

The set of nodes $V$ and the sets of $\operatorname{arcs} A^{J}$ and $A^{L}$ are defined respectively in (13), (14), and (15).

\section{Decision variables}

$f_{i, j, t}$ : flow on job arc $(i, j) \in A^{J}$ in arc-flow structure $t$. The variable indicates the quantity of batches created with position $i$ occupied by jobs with size $j-i$.

$y_{i, t}$ : flow on the loss arc $(i, B) \in A^{L}$ in arc-flow structure $t$.

$v_{t}$ : flow on the feedback arc in arc-flow structure $t$. The variable indicates the number of batches required with processing time $P_{t}$.

$z_{\ell, t}$ : number of jobs with size $S_{\ell}$, not allocated in the batches with processing time smaller than or equal to $P_{t}$. Theses jobs are allowed to be allocated in the batches with processing time $P_{t+1}$.

$$
\begin{aligned}
& \text { (FLOW) } \min \sum_{\forall t \in T} P_{t} \cdot v_{t} \\
& \sum_{(0, i) \in A^{J}} f_{0, i, t}-v_{t}=0 \\
& \sum_{(i, j) \in A^{J}} f_{i, j, t}-\sum_{(j, i) \in A^{J}} f_{j, i, t}-y_{j, t}=0, \\
& \sum_{(i, B) \in A^{J}} f_{i, B, t}+\sum_{(i, B) \in A^{L}} y_{i, t}-v_{t}=0, \\
& N T_{\ell, t}-\sum_{\substack{(i, j) \in A^{J}: \\
j-i=S_{\ell}}} f_{i, j, t}= \begin{cases}z_{\ell, t}, & \text { if } t=1, \\
-z_{\ell, t-1}, & \text { if } t=\delta, \\
z_{\ell, t}-z_{\ell, t-1}, & \text { if } 1<t<\delta,\end{cases} \\
& f_{i, j, t} \leq \min \left(N J_{t}, N T_{j-i, t}^{+}\right), f_{i, j, t} \in \mathbb{Z}, \\
& v_{t} \leq N J_{t}, v_{t} \in \mathbb{Z} \text {, } \\
& y_{i, t} \leq N J_{t}, y_{i, t} \in \mathbb{Z}, \\
& z_{\ell, t} \leq N T_{\ell, t}^{+}, z_{\ell, t} \in \mathbb{Z}, \\
& t \in T \\
& t \in T, j \in\{1, \ldots, B-1\} \\
& t \in T \\
& t \in T, \ell \in\{1, \ldots, \theta\} \\
& t \in T,(i, j) \in A^{J} \\
& t \in T \\
& t \in T,(i, B) \in A^{L} \\
& t \in T: t<\delta, \ell \in\{1, \ldots, \theta\}
\end{aligned}
$$

The objective function (17) minimizes the makespan. Constraints (18), (19), and (20) ensure the flow conservation in node 0 , nodes $1, \ldots, B-1$, and node $B$, respectively . Constraints (21) ensure that all jobs are assigned and also control the number of jobs of each size that are assigned to each arc-flow structure. Constraints (22), (23), (24) and (25) define the domains of the variables and their respective upper bounds, defined by Rules 3-4.

\section{Computational results}

In this section, we discuss the numerical experiments where we compare the new arc-flow formulation (FLOW) for problem $1\left|s_{j}, B\right| C_{\max }$, to the formulations from the literature, (MILP 1$)$ and $\left(\mathrm{MILP}_{1}^{+}\right)$. Three sets of test instances were considered in our experiments. The first and second sets were respectively created by the authors of [4] and [29], who kindly made them available. The third set was created in this work, with instances randomly generated. The selection of the parameters for generating these instances aimed at making them challenging for our formulation. The experiments

(C) 2021. This manuscript version is made available under the CC BY-NC-ND 4.0 license. 
were executed on a computer with a $2.70 \mathrm{GHz}$ Intel Quad-Core Xeon E5-2697 v2 processor and 64GB of RAM, using CPLEX, version 12.7.1.0. The computational time was limited to 1800 seconds for each run. The computational time to construct the graphs for the arc-flow formulation is included in the computational time for all tables.

\subsection{Instances from Chen et al. (2011)}

The first set of test instances for problem $1\left|s_{j}, B\right| C_{\max }$ is the same one considered in [4]. To generate the instances used in our tests, six different numbers of jobs $\left(n_{J}\right)$ were considered, as well as two intervals of integers $\left(p_{1}, p_{2}\right)$, from which the processing times were randomly selected, and three intervals of integers $\left(s_{1}, s_{2}, s_{3}\right)$, from which the sizes of the jobs were randomly selected. Considering the parameters shown in Table 3 , instances of $36(6 \times 2 \times 3)$ configurations were tested. For each configuration, 100 instances were generated. We solved the test instances with CPLEX, configured to run in only one thread.

\begin{tabular}{cccc}
\hline $\begin{array}{c}\text { Number of jobs } \\
n_{J}\end{array}$ & $\begin{array}{c}\text { Processing time } \\
p_{j}\end{array}$ & $\begin{array}{c}\text { Job size } \\
s_{j}\end{array}$ & $\begin{array}{c}\text { Machine capacity } \\
B\end{array}$ \\
\hline $10,20,50$, & $p_{1}:[1,10]$ & $s_{1}:[1,10]$ & $B=10$ \\
$100,300,500$ & $p_{2}:[1,20]$ & $s_{2}:[2,4]$ & \\
& & $s_{3}:[4,8]$ & \\
\hline
\end{tabular}

Table 3: Parameter setting for instances from [4].

The following statistics were considered in our comparative analysis: the computational time of CPLEX in seconds $(T(s))$, the makespan corresponding to the best solution obtained by CPLEX $\left(C_{\max }\right)$, the duality gap of CPLEX at the end of its execution (Gap), and the number of instances for which CPLEX finds the optimal solution (\#O).

We present in Table 4 comparison results among the three formulations for problem $1\left|s_{j}, B\right| C_{\max }$. For a quicker overview of the results in the table we mark bold the best solution between (MILP $\left.{ }_{1}^{+}\right)$ and $(\mathrm{FLOW})\left(\left(\mathrm{MILP}_{1}\right)\right.$ is always the worst), i.e., the best $C_{\max }$, Gap and \#O. If they are the same, none is marked bold. Concerning the time, it is marked bold only if it is smaller and corresponds to the best solution (or one of the best solutions, if they are the same). The same bold mark was used in Tables 7 and $10-14$, presented in the remainder of this section. All values presented in these tables are average results computed over the instances of the same configuration. We note that if some instances in a group are not solved to optimality, the average computational time shown still considers all the instances, assuming that the time for the unsolved instances is equal to the 1800 second time limit.

The comparative tests clearly show that formulation (FLOW) is far superior to (MILP 1 ) and $\left(\mathrm{MILP}_{1}^{+}\right)$for these instances, especially when the number of jobs increases. This is the first time optimal solutions are shown for all these instances, with up to 500 jobs. Considering the small instances, with 50 jobs or less, we see that $\left(\mathrm{MILP}_{1}^{+}\right)$can solve some of the instances in less computational time than (FLOW), but the difference between times is always a fraction of a second. Additionally, the duality gaps shown for $\left(\mathrm{MILP}_{1}\right)$ reveal the difficulty in obtaining good lower bounds with this formulation. This difficulty is reduced with the use of $\left(\mathrm{MILP}_{1}^{+}\right)$, but only (FLOW) can show results with no gap for any instances with 50 jobs or more. With formulation (FLOW), we were able to prove the optimality for $86 \%$ of the instances in less than 1 second, while with models $\left(\mathrm{MILP}_{1}^{+}\right)$and $\left(\mathrm{MILP}_{1}\right)$, we prove optimality for $56.75 \%$ and $17.28 \%$ of the instances, respectively. Furthermore, the total computational time spent on our 3600 test instances, was approximately 45 minutes when using $(\mathrm{FLOW}), 16$ days when using $\left(\mathrm{MILP}_{1}^{+}\right)$, and 50 days when using $\left(\mathrm{MILP}_{1}\right)$.

Unlike what occurs with models $\left(\mathrm{MILP}_{1}\right)$ and $\left(\mathrm{MILP}_{1}^{+}\right)$, the number of variables in $(\mathrm{FLOW})$ does not increase when the number of jobs increases. Moreover, the flow graph does not change in this case. Only the bounds on the variables change. The flow graphs of two distinct instances will be the same if the settings in the parameters Processing Time, Job Size, and Machine Capacity are the same. In fact, this is a very important characteristic of the flow approach. We finally note that

(C) 2021. This manuscript version is made available under the CC BY-NC-ND 4.0 license. 


\begin{tabular}{|c|c|c|c|c|c|c|c|c|c|c|c|c|c|}
\hline \multirow{2}{*}{\multicolumn{2}{|c|}{$\begin{array}{c}\text { Instance } \\
\text { Jobs Type }\end{array}$}} & \multicolumn{4}{|c|}{$\left(\mathrm{MILP}_{1}\right)$} & \multicolumn{4}{|c|}{$\left(\mathrm{MILP}_{1}^{+}\right)$} & \multicolumn{4}{|c|}{$(\mathrm{FLOW})$} \\
\hline & & $T(s)$ & $C_{\max }$ & Gap & $\# \mathrm{O}$ & $T(s)$ & $C_{\max }$ & Gap & $\# \mathrm{O}$ & $T(s)$ & $C_{\max }$ & Gap & $\# \mathrm{O}$ \\
\hline \multicolumn{14}{|c|}{ Instances with $p_{1}=[1,10]$} \\
\hline 10 & $p_{1} s_{1}$ & 0.08 & 36.86 & 0.00 & 100 & \begin{tabular}{|l}
0.00 \\
\end{tabular} & 36.86 & 0.00 & 100 & 0.01 & 36.86 & 0.00 & 100 \\
\hline 10 & $p_{1} s_{2}$ & 0.04 & 20.38 & 0.00 & 100 & 0.01 & 20.38 & 0.00 & 100 & 0.02 & 20.38 & 0.00 & 100 \\
\hline 10 & $p_{1} s_{3}$ & 0.10 & 43.79 & 0.00 & 100 & 0.00 & 43.79 & 0.00 & 100 & 0.00 & 43.79 & 0.00 & 100 \\
\hline 20 & $p_{1} s_{1}$ & 42.62 & 68.07 & 0.06 & 99 & 0.01 & 68.07 & 0.00 & 100 & 0.03 & 68.07 & 0.00 & 100 \\
\hline 20 & $p_{1} s_{2}$ & 300.49 & 37.13 & 1.17 & 92 & 0.09 & 37.13 & 0.00 & 100 & 0.06 & 37.13 & 0.00 & 100 \\
\hline 20 & $p_{1} s_{3}$ & 24.37 & 83.69 & 0.03 & 99 & 0.00 & 83.69 & 0.00 & 100 & 0.01 & 83.69 & 0.00 & 100 \\
\hline 50 & $p_{1} s_{1}$ & 1618.35 & 164.17 & 7.83 & 15 & 0.84 & 164.08 & 0.00 & 100 & 0.19 & 164.08 & 0.00 & 100 \\
\hline 50 & $p_{1} s_{2}$ & 1800.00 & 87.94 & 66.65 & 0 & 317.12 & 87.39 & 0.20 & 88 & 0.36 & 87.39 & 0.00 & 100 \\
\hline 50 & $p_{1} s_{3}$ & 1649.45 & 202.03 & 14.17 & 13 & 0.02 & 202.03 & 0.00 & 100 & 0.02 & 202.03 & 0.00 & 100 \\
\hline 100 & $p_{1} s_{1}$ & 1800.00 & 325.38 & 73.99 & 0 & 70.24 & 318.99 & 0.02 & 97 & 0.17 & 318.99 & 0.00 & 100 \\
\hline 100 & $p_{1} s_{2}$ & 1800.00 & 183.78 & 88.82 & 0 & 1689.67 & 170.58 & 1.41 & 7 & 0.61 & 170.58 & 0.00 & 100 \\
\hline 100 & $p_{1} s_{3}$ & 1800.00 & 401.70 & 83.99 & 0 & 0.11 & 396.96 & 0.00 & 100 & 0.02 & 396.96 & 0.00 & 100 \\
\hline 300 & $p_{1} s_{1}$ & 1800.00 & 1832.31 & 99.38 & 0 & 467.18 & 928.64 & 0.05 & 76 & 0.23 & 928.63 & 0.00 & 100 \\
\hline 300 & $p_{1} s_{2}$ & 1800.00 & 2508.79 & 99.53 & 0 & 1800.00 & 496.07 & 0.86 & 0 & 1.04 & 495.66 & 0.00 & 100 \\
\hline 300 & $p_{1} s_{3}$ & 1800.00 & 1999.85 & 99.44 & 0 & 20.40 & 1174.46 & 0.0009 & 99 & 0.10 & 1174.46 & 0.00 & 100 \\
\hline 500 & $p_{1} s_{1}$ & 1800.00 & 3112.85 & 99.77 & 0 & 825.10 & 1544.31 & 0.05 & 59 & 0.18 & 1544.30 & 0.00 & 100 \\
\hline 500 & $p_{1} s_{2}$ & 1800.00 & 4970.07 & 99.80 & 0 & 1800.00 & 832.43 & 0.72 & 0 & 0.98 & 831.04 & 0.00 & 100 \\
\hline 500 & $p_{1} s_{3}$ & 1800.00 & 3347.23 & 99.88 & 0 & 34.39 & 1949.76 & 0.0005 & 99 & 0.02 & 1949.76 & 0.00 & 100 \\
\hline \multicolumn{2}{|c|}{ Avg. } & 1201.97 & 1079.22 & 51.92 & 34.33 & 390.29 & 475.31 & 0.18 & 79.17 & $\mid 0.22$ & 475.21 & 0.00 & 100.00 \\
\hline \multicolumn{14}{|c|}{ Instances with $p_{2}=[1,20]$} \\
\hline 10 & $p_{2} s_{1}$ & 0.07 & 67.62 & 0.00 & 100 & 0.00 & 67.62 & 0.00 & 100 & 0.02 & 67.62 & 0.00 & 100 \\
\hline 10 & $p_{2} s_{2}$ & 0.04 & 40.22 & 0.00 & 100 & 0.02 & 40.22 & 0.00 & 100 & 0.03 & 40.22 & 0.00 & 100 \\
\hline 10 & $p_{2} s_{3}$ & 0.09 & 81.05 & 0.00 & 100 & 0.00 & 81.05 & 0.00 & 100 & 0.01 & 81.05 & 0.00 & 100 \\
\hline 20 & $p_{2} s_{1}$ & 22.35 & 133.09 & 0.01 & 99 & 0.01 & 133.09 & 0.00 & 100 & 0.09 & 133.09 & 0.00 & 100 \\
\hline 20 & $p_{2} s_{2}$ & 215.7 & 72.88 & 0.96 & 94 & 0.09 & 72.88 & 0.00 & 100 & 0.13 & 72.88 & 0.00 & 100 \\
\hline 20 & $p_{2} s_{3}$ & 16.49 & 159.11 & 0.00 & 100 & 0.00 & 159.11 & 0.00 & 100 & 0.01 & 159.11 & 0.00 & 100 \\
\hline 50 & $p_{2} s_{1}$ & 1596.95 & 314.76 & 9.40 & 15 & 0.38 & 314.57 & 0.00 & 100 & 0.62 & 314.57 & 0.00 & 100 \\
\hline 50 & $p_{2} s_{2}$ & 1800.00 & 169.93 & 65.57 & 0 & 178.70 & 168.11 & 0.06 & 94 & 1.81 & 168.11 & 0.00 & 100 \\
\hline 50 & $p_{2} s_{3}$ & 1701.53 & 384.15 & 15.56 & 8 & 0.02 & 384.13 & 0.00 & 100 & 0.02 & 384.13 & 0.00 & 100 \\
\hline 100 & $p_{2} s_{1}$ & 1800.00 & 622.84 & 76.62 & 0 & 33.35 & 610.64 & 0.01 & 99 & 0.87 & 610.64 & 0.00 & 100 \\
\hline 100 & $p_{2} s_{2}$ & 1800.00 & 357.69 & 89.35 & 0 & 1717.84 & 326.14 & 0.99 & 6 & 4.71 & 326.11 & 0.00 & 100 \\
\hline 100 & $p_{2} s_{3}$ & 1800.00 & 775.50 & 83.96 & 0 & 0.10 & 766.91 & 0.00 & 100 & 0.04 & 766.91 & 0.00 & 100 \\
\hline 300 & $p_{2} s_{1}$ & 1800.00 & 3979.09 & 99.42 & 0 & 329.03 & 1793.54 & 0.02 & 83 & 0.96 & 1793.52 & 0.00 & 100 \\
\hline 300 & $p_{2} s_{2}$ & 1800.00 & 5925.99 & 99.66 & 0 & 1800.00 & 965.42 & 1.08 & 0 & 6.56 & 962.77 & 0.00 & 100 \\
\hline 300 & $p_{2} s_{3}$ & 1800.00 & 3983.88 & 99.43 & 0 & 21.78 & 2247.39 & 0.0005 & 99 & 0.17 & 2247.39 & 0.00 & 100 \\
\hline 500 & $p_{2} s_{1}$ & 1800.00 & 6264.18 & 99.92 & 0 & 780.62 & 2964.62 & 0.03 & 63 & 0.89 & 2964.57 & 0.00 & 100 \\
\hline 500 & $p_{2} s_{2}$ & 1800.00 & 9937.42 & 99.94 & 0 & 1800.00 & 1592.72 & 0.86 & 0 & 5.66 & 1587.50 & 0.00 & 100 \\
\hline 500 & $p_{2} s_{3}$ & 1800.00 & 6822.02 & 99.92 & 0 & 97.16 & 3701.79 & 0.0013 & 96 & 0.13 & 3701.79 & 0.00 & 100 \\
\hline \multicolumn{2}{|c|}{ Avg. } & 1197.40 & 2227.30 & 52.21 & 34.22 & 375.51 & 910.55 & 0.17 & 80.00 & 1.26 & 910.11 & 0.00 & 100.00 \\
\hline
\end{tabular}

Table 4: Computational results for the instances available by [4] for the $1\left|s_{j} . B\right| C_{\max }$ problem.

(C) 2021. This manuscript version is made available under the CC BY-NC-ND 4.0 license. 
the computational time to construct the graphs for the flow formulation was included in these times. However, we should mention that the maximum time to construct a graph for any instance in these experiments was 0.008 second.

In Table 5 we also analyze the quality of the formulations $\left(\mathrm{MILP}_{1}^{+}\right)$and $(\mathrm{FLOW})$ by comparing the solution costs of their linear relaxations, and verifying how tight the bounds generated by them are. The following statistics are shown: the makespan corresponding to the optimal solution of the instance previously calculated $\left(C_{\max } *\right)$, the makespan corresponding to the solution costs of the relaxations $\left(C_{\max }\right)$, the gap given by: Gap $=\left(C_{\max } *-C_{\max }\right) / C_{\max }$, the number of simplex iterations (Iter.), and the computational time in seconds $(T(s))$. We mark bold the best Gap and the best $C_{\max }$ between the two formulations. The time and number of iterations are also marked bold if they are smaller and correspond to the best Gap or the best $C_{\max }$.

We notice that the linear relaxation of (FLOW) is much tighter than the linear relaxation of $\left(\mathrm{MILP}_{1}^{+}\right)$for most instances, especially when the number of jobs increases. For instances of type $s_{2}$, the solutions of the linear relaxations are very close to each other, but (FLOW) is slightly better in this case. Another critical point is that (FLOW) presents lower computational time for solving the linear relaxations. For instances with 500 jobs and type $p_{2} s_{2}$, the time for linear relaxation of (FLOW) is $0.66 \%$ of the time for the linear relaxation of $\left(\mathrm{MILP}_{1}^{+}\right)$. This time difference tends to increase when the number of jobs increases. These results give us some insight into why (FLOW) overcomes $\left(\mathrm{MILP}_{1}^{+}\right)$.

The results in Tables 4 and 5 show that instances of configuration $s_{2}$ require more computational time and are more difficult when compared to the other instances for all formulations. The reason for this is the small sizes of the jobs when compared to the machine capacity, which allows more combinations of assignment to a batch. In this way, the number of feasible solutions for instances of type $s_{2}$ is greater than for the other instances.

With the new formulation (FLOW), it is possible to find the optimal solution of all the instances proposed by [4] with good computational times.

\subsection{Instances from Muter (2020)}

The second set of test instances for problem $1\left|s_{j}, B\right| C_{\max }$ is the same one considered in the recent work [29]. To generate these instances, four different numbers of jobs $\left(n_{J}\right)$ were considered, as well as three intervals of integers $\left(p_{1}, p_{2}, p_{3}\right)$, from which the processing times were randomly selected, and three intervals of integers $\left(s_{1}, s_{2}, s_{3}\right)$, from which the sizes of the jobs were randomly selected. Considering the parameters shown in Table 4.2 , instances of $36(4 \times 3 \times 3)$ configurations were tested. For each configuration, 20 instances were generated. We solved the instances with CPLEX configured in the default settings, with multi-threading enabled. We emphasize that we use in these tests similar configuration to the one used in [29].

We keep the notation used by the author to represent the instances in Table 7 , where $n_{j}$ is the number of jobs, and $\bar{s}$ and $\bar{p}$ are the maximum sizes and processing times of the jobs in the interval. The same statistics presented in Table 4 are presented in Table 7 comparing (MILP ${ }_{1}^{+}$) and (FLOW) on this other set of instances.

For the second set of instances we have a similar analysis to the one obtained for the first set. Although most instances were solved to optimality by both formulations, on the most difficult instances, (FLOW) still performs better. These are specifically all the instances with $\bar{s}=4$ and $n_{J}$ equal to 50 or 100 . Considering the small instances, with 20 jobs or less, we see that $\left(\mathrm{MILP}_{1}^{+}\right)$ can solve almost all instances in less computational time than (FLOW), but the difference between times is a fraction of a second. Considering instances with 50 or 100 jobs, we see that the average time used by (FLOW) is smaller than the average time used by $\left(\mathrm{MILP}_{1}^{+}\right)$. The average time to solve all instances was 121.73 seconds for $\left(\mathrm{MILP}_{1}^{+}\right)$, compared to only 14.41 seconds for (FLOW). However, we should note that the difficulty for (FLOW) increases when the processing time increases, which is expected. With formulation (FLOW), we were able to prove the optimality for $99.6 \%$ of the instances, while with $\left(\mathrm{MILP}_{1}^{+}\right)$we prove optimality for $93.9 \%$ of them. The longest graph construction time of the arc-flow formulation for these instances is $0.005 \mathrm{~s}$.

In [29], a column-and-cut generation CCG algorithm is applied to all the instances of the test set

(C) 2021. This manuscript version is made available under the CC BY-NC-ND 4.0 license. 


\begin{tabular}{|c|c|c|c|c|c|c|c|c|c|c|}
\hline \multicolumn{3}{|c|}{ Instance } & \multicolumn{4}{|c|}{$\left(\right.$ MILP $\left._{1}^{+}\right)$- Relax } & \multicolumn{4}{|c|}{ (FLOW) - Relax } \\
\hline Jobs & Type & $C_{\text {max }} *$ & $C_{\max }$ & Gap & Iter. & $T(s)$ & $C_{\max }$ & Gap & Iter. & $T(s)$ \\
\hline \multicolumn{11}{|c|}{ Instances with $p_{1}=[1,10]$} \\
\hline 10 & $p_{1} s_{1}$ & 36.86 & 32.34 & 12.26 & 29.41 & 0.00 & 34.41 & 6.66 & 79.52 & 0.00 \\
\hline 10 & $p_{1} s_{2}$ & 20.38 & 18.61 & 8.70 & 36.66 & 0.00 & 16.79 & 17.61 & 82.88 & 0.00 \\
\hline 10 & $p_{1} s_{3}$ & 43.79 & 34.82 & 20.49 & 34.09 & 0.00 & 41.72 & 4.74 & 14.77 & 0.00 \\
\hline 20 & $p_{1} s_{1}$ & 68.07 & 61.42 & 9.77 & 80.21 & 0.00 & 65.34 & 4.02 & 181.54 & 0.00 \\
\hline 20 & $p_{1} s_{2}$ & 37.13 & 34.54 & 6.99 & 103.60 & 0.00 & 33.38 & 10.11 & 133.90 & 0.00 \\
\hline 20 & $p_{1} s_{3}$ & 83.69 & 66.80 & 20.18 & 96.61 & 0.00 & 81.49 & 2.63 & 26.04 & 0.00 \\
\hline 50 & $p_{1} s_{1}$ & 164.08 & 151.94 & 7.40 & 330.65 & 0.01 & 161.03 & 1.86 & 318.89 & 0.01 \\
\hline 50 & $p_{1} s_{2}$ & 87.39 & 83.07 & 4.94 & 341.59 & 0.01 & 83.15 & 4.85 & 168.99 & 0.00 \\
\hline 50 & $p_{1} s_{3}$ & 202.03 & 165.65 & 18.01 & 385.43 & 0.01 & 199.71 & 1.15 & 42.95 & 0.00 \\
\hline 100 & $p_{1} s_{1}$ & 318.99 & 302.63 & 5.13 & 675.16 & 0.04 & 315.98 & 0.94 & 386.88 & 0.01 \\
\hline 100 & $p_{1} s_{2}$ & 170.58 & 165.68 & 2.87 & 720.13 & 0.05 & 165.99 & 2.69 & 178.51 & 0.00 \\
\hline 100 & $p_{1} s_{3}$ & 396.96 & 328.82 & 17.17 & 742.44 & 0.05 & 394.65 & 0.58 & 56.13 & 0.00 \\
\hline 300 & $p_{1} s_{1}$ & 928.63 & 902.86 & 2.77 & 2729.99 & 0.36 & 925.87 & 0.30 & 467.62 & 0.01 \\
\hline 300 & $p_{1} s_{2}$ & 495.66 & 490.81 & 0.98 & 3186.63 & 0.44 & 490.85 & 0.97 & 180.92 & 0.01 \\
\hline 300 & $p_{1} s_{3}$ & 1174.46 & 988.13 & 15.87 & 3043.30 & 0.40 & 1172.12 & 0.20 & 62.20 & 0.00 \\
\hline 500 & $p_{1} s_{1}$ & 1544.30 & 1513.43 & 2.00 & 4673.94 & 1.19 & 1541.61 & 0.17 & 480.35 & 0.01 \\
\hline 500 & $p_{1} s_{2}$ & 831.04 & 826.12 & 0.59 & 5387.19 & 1.64 & 826.12 & 0.59 & 180.45 & 0.01 \\
\hline 500 & $p_{1} s_{3}$ & 1949.76 & 1645.82 & 15.59 & 5354.30 & 1.47 & 1947.47 & 0.12 & 61.54 & 0.00 \\
\hline \multicolumn{2}{|c|}{ Avg. } & 475.21 & 434.08 & 9.54 & 1552.85 & 0.32 & 472.09 & 3.34 & 172.45 & 0.003 \\
\hline \multicolumn{11}{|c|}{ Instances with $p_{2}=[1,20]$} \\
\hline 10 & $p_{2} s_{1}$ & 67.62 & 59.67 & 11.75 & 30.16 & 0.00 & 62.33 & 7.82 & 109.14 & 0.00 \\
\hline 10 & $p_{2} s_{2}$ & 40.22 & 36.55 & 9.14 & 37.14 & 0.00 & 32.69 & 18.72 & 101.74 & 0.00 \\
\hline 10 & $p_{2} s_{3}$ & 81.05 & 64.90 & 19.93 & 33.71 & 0.00 & 77.00 & 5.00 & 18.71 & 0.00 \\
\hline 20 & $p_{2} s_{1}$ & 133.09 & 119.97 & 9.86 & 84.59 & 0.00 & 128.09 & 3.75 & 253.47 & 0.01 \\
\hline 20 & $p_{2} s_{2}$ & 72.88 & 68.24 & 6.37 & 105.44 & 0.00 & 65.23 & 10.50 & 181.63 & 0.00 \\
\hline 20 & $p_{2} s_{3}$ & 159.11 & 129.26 & 18.76 & 97.66 & 0.00 & 154.68 & 2.78 & 38.11 & 0.00 \\
\hline 50 & $p_{2} s_{1}$ & 314.57 & 291.85 & 7.22 & 331.51 & 0.01 & 309.22 & 1.70 & 566.30 & 0.02 \\
\hline 50 & $p_{2} s_{2}$ & 168.11 & 161.19 & 4.12 & 352.33 & 0.01 & 159.89 & 4.89 & 295.78 & 0.00 \\
\hline 50 & $p_{2} s_{3}$ & 384.13 & 313.80 & 18.31 & 374.71 & 0.01 & 379.53 & 1.20 & 73.38 & 0.00 \\
\hline 100 & $p_{2} s_{1}$ & 610.64 & 579.74 & 5.06 & 693.34 & 0.05 & 605.19 & 0.89 & 782.51 & 0.02 \\
\hline 100 & $p_{2} s_{2}$ & 326.11 & 316.77 & 2.86 & 742.66 & 0.05 & 317.20 & 2.73 & 335.60 & 0.01 \\
\hline 100 & $p_{2} s_{3}$ & 766.91 & 633.63 & 17.38 & 783.24 & 0.05 & 762.26 & 0.61 & 102.42 & 0.00 \\
\hline 300 & $p_{2} s_{1}$ & 1793.52 & 1737.65 & 3.11 & 2786.36 & 0.36 & 1788.23 & 0.29 & 963.18 & 0.03 \\
\hline 300 & $p_{2} s_{2}$ & 962.77 & 952.38 & 1.08 & 3181.75 & 0.41 & 952.79 & 1.04 & 357.50 & 0.01 \\
\hline 300 & $p_{2} s_{3}$ & 2247.39 & 1892.29 & 15.80 & 3161.54 & 0.41 & 2242.72 & 0.21 & 135.67 & 0.00 \\
\hline 500 & $p_{2} s_{1}$ & 2964.57 & 2895.60 & 2.33 & 4966.34 & 1.25 & 2959.25 & 0.18 & 1017.03 & 0.04 \\
\hline 500 & $p_{2} s_{2}$ & 1587.50 & 1577.43 & 0.63 & 5496.04 & 1.51 & 1577.56 & 0.63 & 360.63 & 0.01 \\
\hline 500 & $p_{2} s_{3}$ & 3701.79 & 3138.60 & 15.21 & 5664.89 & 1.52 & 3697.31 & 0.12 & 136.55 & 0.00 \\
\hline \multicolumn{2}{|c|}{ Avg. } & 910.11 & 831.64 & 9.38 & 1606.86 & 0.31 & 903.95 & 3.50 & 323.85 & 0.008 \\
\hline
\end{tabular}

Table 5: Comparison between the linear relaxations for (MILP ${ }_{1}^{+}$) and (FLOW) for the $1\left|s_{j}, B\right| C_{\max }$ problem.

(C) 2021. This manuscript version is made available under the CC BY-NC-ND 4.0 license. 


\begin{tabular}{cccc}
\hline $\begin{array}{c}\text { Number of jobs } \\
\left(n_{J}\right)\end{array}$ & $\begin{array}{c}\text { Processing time } \\
\left(p_{j}\right)\end{array}$ & $\begin{array}{c}\text { Job size } \\
\left(s_{j}\right)\end{array}$ & $\begin{array}{c}\text { Machine capacity } \\
(B)\end{array}$ \\
\hline $10,20,50$, & $p_{1}:[1,10]$ & $s_{1}:[1,10]$ & $B=10$ \\
100 & $p_{2}:[1,20]$ & $s_{2}:[2,4]$ & \\
& $p_{3}:[1,100]$ & $s_{3}:[4,8]$ & \\
\hline
\end{tabular}

Table 6: Parameter setting for instances from [29].

\begin{tabular}{|c|c|c|c|c|c|c|c|c|c|c|}
\hline \multicolumn{3}{|c|}{ Instance } & \multicolumn{4}{|c|}{$\left(\mathrm{MILP}_{1}^{+}\right)$} & \multicolumn{4}{|c|}{ (FLOW) } \\
\hline$n_{j}$ & $\bar{s}$ & $\bar{p}$ & $T(s)$ & $C_{\max }$ & Gap & $\# \mathrm{O}$ & $T(s)$ & $C_{\max }$ & Gap & $\# \mathrm{O}$ \\
\hline 10 & 4 & 10 & 0.05 & 21.45 & 0.00 & 20 & 0.08 & 21.45 & 0.00 & 20 \\
\hline 10 & 4 & 20 & 0.05 & 39.15 & 0.00 & 20 & 0.10 & 39.15 & 0.00 & 20 \\
\hline 10 & 4 & 100 & 0.06 & 202.35 & 0.00 & 20 & 0.11 & 202.35 & 0.00 & 20 \\
\hline 10 & 8 & 10 & 0.01 & 44.85 & 0.00 & 20 & 0.02 & 44.85 & 0.00 & 20 \\
\hline 10 & 8 & 20 & 0.02 & 78.25 & 0.00 & 20 & 0.03 & 78.25 & 0.00 & 20 \\
\hline 10 & 8 & 100 & 0.01 & 414.10 & 0.00 & 20 & 0.03 & 414.10 & 0.00 & 20 \\
\hline 10 & 10 & 10 & 0.03 & 34.25 & 0.00 & 20 & 0.05 & 34.25 & 0.00 & 20 \\
\hline 10 & 10 & 20 & 0.02 & 71.35 & 0.00 & 20 & 0.05 & 71.35 & 0.00 & 20 \\
\hline 10 & 10 & 100 & 0.02 & 333.85 & 0.00 & 20 & 0.06 & 33 & 0.00 & 20 \\
\hline 20 & 4 & 10 & 10 & 37.35 & 0.00 & 20 & 0.09 & & 0.00 & 20 \\
\hline 20 & 4 & 20 & 0.12 & 72.90 & 0.00 & 20 & 16 & 72.90 & 0.00 & 20 \\
\hline 20 & 4 & 100 & 0.13 & 357.95 & 0.00 & 20 & 0.35 & 357.95 & 0.00 & 20 \\
\hline 20 & 8 & 10 & 0.02 & 82.65 & 0.00 & 20 & 0.04 & 82.65 & 0.00 & 20 \\
\hline 20 & 8 & 20 & 0.02 & 159.05 & 0.00 & 20 & 0.04 & & 0.00 & 20 \\
\hline 20 & 8 & 100 & 04 & 811. & 0.00 & 20 & & & 00 & 20 \\
\hline 20 & 10 & 10 & 0.04 & 66.85 & 0.00 & 20 & .07 & 66. & 0.00 & 20 \\
\hline 20 & 10 & 20 & 0.04 & 123.45 & 0.00 & 20 & 0.10 & 123.45 & 0.00 & 20 \\
\hline 20 & 10 & 100 & 0.06 & 639.95 & 0.00 & 20 & 0.21 & 639.95 & 0.00 & 20 \\
\hline 50 & 4 & 10 & 55.52 & 88.30 & 0.00 & 20 & 0.31 & 88.30 & 0.00 & 20 \\
\hline 50 & 4 & 20 & 13.30 & 171.95 & 0.00 & 20 & 0.96 & 171. & 0.00 & 20 \\
\hline 50 & 4 & 100 & 20.39 & 819 & 0.00 & 20 & & & 0.00 & 20 \\
\hline 50 & 8 & 10 & 0.07 & 205.05 & 0.00 & 20 & 0.05 & 205.05 & 0.00 & 20 \\
\hline 50 & 8 & 20 & 0.04 & 397.80 & 0.00 & 20 & 0.06 & 397.80 & 0.00 & 20 \\
\hline 50 & 8 & 100 & 0.06 & 1839.65 & 0.00 & 20 & 0.09 & 1839.65 & 0.00 & 20 \\
\hline 50 & 10 & 10 & 0.15 & 163.70 & 0.00 & 20 & 0.11 & 163.70 & 0.00 & 20 \\
\hline 50 & 10 & 20 & 0.17 & 316.50 & 0.00 & 20 & 0.24 & 316.50 & 0.00 & 20 \\
\hline 50 & 10 & 100 & 0.19 & 1580.2 & 0.00 & 20 & .95 & 1580. & 0.00 & 20 \\
\hline 100 & 4 & 10 & 1688.21 & 172.50 & 1.04 & 3 & 0.34 & 172.50 & 0.00 & 20 \\
\hline 100 & 4 & 20 & 1670.83 & 319.50 & 0.90 & 2 & 2.50 & 319.50 & 0.00 & 20 \\
\hline 100 & 4 & 100 & 928.37 & 1555.40 & 0.29 & 11 & 499.95 & 1555.35 & 0.07 & 17 \\
\hline 100 & 8 & 10 & 0.15 & 399.35 & 0.00 & 20 & 0.06 & 399.35 & 0.00 & 20 \\
\hline 100 & 8 & 20 & 0.17 & 766.75 & 0.00 & 20 & 0.08 & 766.75 & 0.00 & 20 \\
\hline 100 & 8 & 100 & 0.16 & 3808.15 & 0.00 & 20 & 0.17 & 3808.15 & 0.00 & 20 \\
\hline 100 & 10 & 10 & 1.71 & 312.50 & 0.00 & 20 & 0.19 & 312.50 & 0.00 & 20 \\
\hline 100 & 10 & 20 & 0.97 & 597.85 & 0.00 & 20 & 0.29 & 597.85 & 0.00 & 20 \\
\hline 100 & 10 & 100 & 0.86 & 2989.95 & 0.00 & 20 & 3.44 & 2989.95 & 0.00 & 20 \\
\hline Avg & $5 . / \mathrm{s}$ & Sum. & 121.73 & 558.214 & 0.06 & 676 & 14.41 & 558.213 & 0.002 & 717 \\
\hline
\end{tabular}

Table 7: Computational results for the instances available by [29] for the $1\left|s_{j} . B\right| C_{\max }$ problem.

(C) 2021. This manuscript version is made available under the CC BY-NC-ND 4.0 license. 
considered in Table 7 that could not be solved to optimality in 300 seconds when using formulation $\left(\mathrm{MILP}_{1}^{+}\right)$. The results presented in the paper show that the total number of instances solved increase from 676 to 694, still less than the 717 instances solved by (FLOW). The experiments reported in [29] were conducted on the nodes of a computing cluster, each with two Intel E5- 2650 v2 CPUs (16 cores in total) running at $2.60 \mathrm{GHz}$. The CCG algorithm was also solved under a time limit of 1800 seconds.

\subsection{New instances proposed}

From the computational tests with the instances proposed by [4], we notice that these instances are not challenging enough for (FLOW). We illustrate the application of our approach to instances with a large number of jobs, where multiple jobs have equal size and processing time. We created new instances where the number of jobs goes up to 100 million, but the intervals from which the processing times and sizes are selected, were maintained respectively, as $p_{2}$ and $s_{2}$. We aim at emphasizing here how the formulation is particularly suitable for this type of instance. Table 8 shows the computational results obtained. With (FLOW), we could find the optimum solution of all the instances with up to 100 million jobs in no more than 6.05 seconds. We note that column "Construction Time" in Table 8 considers the times to read the data of the instance, sort the jobs and create the parameters to the model, i.e., to construct the graph $G$. The algorithm to construct $G$ is polynomial in the number of jobs, but the construction phase can take longer than the resolution of the problem, when the number of jobs increases. Although the construction time increases with the number of jobs, even when added to the solution time of model (FLOW), it leads to very small times to solve instances of this particular type, when comparing to other formulations from the literature. The column "Nodes" show the number of nodes required by CPLEX to solve the problem.

Table 8: Computational results for large random instances for the $1\left|s_{j}, B\right| C_{\max }$ problem.

\begin{tabular}{rlrrrrr}
\hline \multicolumn{2}{c}{ Instance } & \multicolumn{5}{c}{$($ FLOW $)$} \\
\cline { 3 - 7 } Jobs & Type & $T(s)$ & \multicolumn{1}{c}{$C_{\max }$} & Gap & Nodes & Construction Time $(s)$ \\
\hline 1000 & $p_{2} s_{2}$ & 4.90 & 3241 & 0.00 & 8043 & 0.03 \\
10000 & $p_{2} s_{2}$ & 3.66 & 31719 & 0.00 & 3625 & 0.16 \\
100000 & $p_{2} s_{2}$ & 3.22 & 314945 & 0.00 & 4668 & 1.44 \\
1000000 & $p_{2} s_{2}$ & 2.80 & 3152697 & 0.00 & 3011 & 14.24 \\
10000000 & $p_{2} s_{2}$ & 6.05 & 31495193 & 0.00 & 17111 & 145.07 \\
100000000 & $p_{2} s_{2}$ & 3.25 & 314996812 & 0.00 & 4325 & 886.14 \\
\hline
\end{tabular}

Also, from the tests with the instances proposed by [29], we can see that high processing times increase the difficulty for the (FLOW) model. Therefore, a third set of test instances for problem $1\left|s_{j}, B\right| C_{\max }$ is proposed in this work, with the parameter setting depicted in Table 9 . The main idea is to generate more difficult instances, specifically for (FLOW), in order to better verify for which instances the model is more effective. For this, we explore the increase in the value of the parameter $B$, and in the size of the intervals from which $p_{j}$ and $s_{j}$ are selected. These increases directly affect the number of variables and constraints in formulation (FLOW). Comparing with the set of instances from [4] this new set of instances considers three larger values for the machine capacity $B$. The processing times are randomly selected from two intervals of integers again, but the second interval is much larger when we have a large number of jobs. This will lead to more distinct values of processing times among the jobs, and therefore, to the necessity of more arc-flow structures to represent the solutions. Finally, job sizes are randomly selected from three intervals of integers, where the ranges are proportional to the size of the machine, for example, the interval $s_{2}:=[0.2 B, 0.4 B]$. Note that when $B=100$, the interval is $[20,40]$, so it keeps the similar characteristics of the instances from [4], in terms of scale.

In total, 1320 instances were generated, 10 for each of the 132 different combinations of number of jobs, range of processing times, range of job sizes, and machine capacity shown in the Table 9 .

(C) 2021. This manuscript version is made available under the CC BY-NC-ND 4.0 license. 
We solved the test instances with CPLEX, configured to run in only one thread. The problem data set described in this section is provided as supplementary material.

\begin{tabular}{lllc}
\hline $\begin{array}{c}\text { Number of jobs } \\
\left(n_{J}\right)\end{array}$ & $\begin{array}{c}\text { Processing time } \\
\left(p_{j}\right)\end{array}$ & \multicolumn{1}{c}{$\begin{array}{c}\text { Jobs size } \\
\left(s_{j}\right)\end{array}$} & $\begin{array}{c}\text { Machine capacity } \\
(B)\end{array}$ \\
\hline & & $s_{1}:[1, B]$ & \\
$10,50,100,500$ & $p_{1}:[1,20]$ & $s_{2}:[0.2 B, 0.4 B]$ & $20,50,100$ \\
1000,5000 & $p_{2}:\left[1, n_{J}\right]$ & $s_{3}:[0.4 B, 0.8 B]$ & \\
\cline { 3 - 4 } & & $s_{1}:[1, B]$ & 500,1000 \\
\hline
\end{tabular}

Table 9: Parameter setting for the new set of instances.

We present in Tables 10-14 comparison results between formulations (MILP ${ }_{1}^{+}$) and (FLOW) for the new set of instances generated. Results marked with "-" indicate that the solver could not find a feasible solution for at least one instance of the group. In this case, besides the number of instances of the group for witch the solver proves optimality, the number of instances for witch the solver can find a feasible solution is also indicated in parentheses in column "\#O".

The comparative tests show that new instances are more difficult for (FLOW), especially when the processing times are selected from interval $p_{2}$, as there are more distinct processing times in this case. In this way, (FLOW) needs to consider many flow structures, one for each processing time. The difference between the instances $p_{1}$ and $p_{2}$, however, are not influencing the number of variables in $\left(\mathrm{MILP}_{1}^{+}\right)$, and the computational results show that this model is more stable when the range of the processing time changes.

$\left(\mathrm{MILP}_{1}^{+}\right)$is superior for instances of type $p_{2}$ especially when the machine capacity increases. The results in Table 11 show that (FLOW) was not able to find even a single integer solution in some cases. This behavior occurs in the worst case scenario for (FLOW), where a large value for the machine capacity is combined with the type of instances $p_{2}$. When $B=100$, the (FLOW) model considers many nodes in the arc-flow structures, which reflects in the computational performance. Another difference between this new set of instances and the instances tested in the previous section is that the range of job sizes varies with the number of jobs. The number of arcs in (FLOW) increases as the number of jobs increases.

Even with the difficulties created with this new set of instances, the (FLOW) model obtained superior results for instances of type $p_{1}$ and capacity $B \leq 100$, especially when the number of jobs increases. Considering the instances of type $p_{1}$ with $\bar{B}$ up to 20 , it was possible to find the optimal solution for all instances. Finally, when $B>500,\left(\mathrm{MILP}_{1}^{+}\right)$shows better results, even when the number of jobs increases, as shown in Table 13. These results show that both models are complementary, that is, there are clearly situations in which each model is superior to the other.

\section{Conclusions and Future Work}

We address a single batch-processing machine scheduling problem. The economic importance of the problem has motivated the investigation of good solution approaches, and its NP-hardness has led the majority of this research to focus on heuristic approaches. We show that by applying a good MILP formulation for this scheduling problem, we can go a step further in the exact resolution of applied problems, having presented optimal solutions for test instances with sizes never considered in the literature by exact methods. We were able to present much better results for a set of benchmark instances from the literature with the formulation proposed, when compared to results obtained with two other formulations from the literature.

We propose an arc-flow based formulation for the problem in which the numbers of variables and constraints do not change when the number of jobs increases. This procedure enabled us to find the optimum solution of an instance with 100 million jobs in 3.25 seconds and define a new threshold for the size of the instances, as the maximum number of jobs previously treated in the literature was 500, using heuristic approaches for instances with the same parameter settings.

(C) 2021. This manuscript version is made available under the CC BY-NC-ND 4.0 license. 


\begin{tabular}{|c|c|c|c|c|c|c|c|c|c|c|}
\hline \multirow{2}{*}{ Jobs } & \multirow{2}{*}{$\begin{array}{l}\text { Instance } \\
\text { Capacity }\end{array}$} & \multirow{2}{*}{ Type } & \multicolumn{4}{|c|}{$\left(\mathrm{MILP}_{1}^{+}\right)$} & \multicolumn{4}{|c|}{ (FLOW) } \\
\hline & & & $T(s)$ & $C_{\max }$ & Gap & $\# \mathrm{O}$ & $T(s)$ & $C_{\max }$ & Gap & \#O \\
\hline \multicolumn{11}{|c|}{ Instances with $p_{1}=[1,20]$ and $B=20$} \\
\hline 10 & 20 & $p_{1} s_{1}$ & 0.01 & 68.50 & 0.00 & 10 & 0.02 & 68.50 & 0.00 & 10 \\
\hline 10 & 20 & $p_{1} s_{2}$ & 0.02 & 42.90 & 0.00 & 10 & 0.09 & 42.90 & 0.00 & 10 \\
\hline 10 & 20 & $p_{1} s_{3}$ & 0.01 & 65.90 & 0.00 & 10 & 0.01 & 65.90 & 0.00 & 10 \\
\hline 50 & 20 & $p_{1} s_{1}$ & 0.38 & 318.10 & 0.00 & 10 & 1.09 & 318.10 & 0.00 & 10 \\
\hline 50 & 20 & $p_{1} s_{2}$ & 300.17 & 175.90 & 0.00 & 10 & 9.11 & 175.90 & 0.00 & 10 \\
\hline 50 & 20 & $p_{1} s_{3}$ & 0.01 & 395.40 & 0.00 & 10 & 0.02 & 395.40 & 0.00 & 10 \\
\hline 100 & 20 & $p_{1} s_{1}$ & 0.80 & 629.50 & 0.00 & 10 & 1.19 & 629.50 & 0.00 & 10 \\
\hline 100 & 20 & $p_{1} s_{2}$ & 1472.37 & 326.30 & 0.86 & 3 & 36.07 & 326.00 & 0.00 & 10 \\
\hline 100 & 20 & $p_{1} s_{3}$ & 0.05 & 801.00 & 0.00 & 10 & 0.03 & 801.00 & 0.00 & 10 \\
\hline 500 & 20 & $p_{1} s_{1}$ & 621.04 & 2839.70 & 0.02 & 8 & 3.50 & 2839.50 & 0.00 & 10 \\
\hline 500 & 20 & $p_{1} s_{2}$ & 1800.00 & 1615.80 & 1.64 & 0 & 23.53 & 1599.90 & 0.00 & 10 \\
\hline 500 & 20 & $p_{1} s_{3}$ & 1.76 & 3841.50 & 0.00 & 10 & 0.06 & 3841.50 & 0.00 & 10 \\
\hline 1000 & 20 & $p_{1} s_{1}$ & 1721.60 & 5727.50 & 0.07 & 1 & 34.55 & 5726.30 & 0.00 & 10 \\
\hline 1000 & 20 & $p_{1} s_{2}$ & 1800.00 & 10428.00 & 109.37 & 0 & 24.62 & 3153.10 & 0.00 & 10 \\
\hline 1000 & 20 & $p_{1} s_{3}$ & 37.78 & 7643.40 & 0.00 & 10 & 0.18 & 7643.40 & 0.00 & 10 \\
\hline 5000 & 20 & $p_{1} s_{1}$ & 1800.00 & 52393.50 & 533.43 & 0 & 3.77 & 27851.60 & 0.00 & 10 \\
\hline 5000 & 20 & $p_{1} s_{2}$ & 1800.00 & 52514.30 & 99.96 & 0 & 19.66 & 15753.50 & 0.00 & 10 \\
\hline 5000 & 20 & $p_{1} s_{3}$ & 1663.61 & 38226.40 & 0.01 & 4 & 0.15 & 38224.30 & 0.00 & 10 \\
\hline \multicolumn{3}{|c|}{ Avg./Sum. } & 723.31 & 9891.87 & 41.41 & 116 & 8.76 & 6080.91 & 0.00 & 180 \\
\hline \multicolumn{11}{|c|}{ Instances with $p_{2}=\left[1, n_{J}\right]$ and $B=20$} \\
\hline 10 & 20 & $p_{2} s_{1}$ & 0.01 & 35.50 & 0.00 & 10 & 0.03 & 35.50 & 0.00 & 10 \\
\hline 10 & 20 & $p_{2} s_{2}$ & 0.02 & 24.00 & 0.00 & 10 & 0.06 & 24.00 & 0.00 & 10 \\
\hline 10 & 20 & $p_{2} s_{3}$ & 0.00 & 48.70 & 0.00 & 10 & 0.01 & 48.70 & 0.00 & 10 \\
\hline 50 & 20 & $p_{2} s_{1}$ & 0.17 & 692.30 & 0.00 & 10 & 7.57 & 692.30 & 0.00 & 10 \\
\hline 50 & 20 & $p_{2} s_{2}$ & 75.73 & 414.10 & 0.00 & 10 & 152.06 & 414.10 & 0.00 & 10 \\
\hline 50 & 20 & $p_{2} s_{3}$ & 0.01 & 995.00 & 0.00 & 10 & 0.04 & 995.00 & 0.00 & 10 \\
\hline 100 & 20 & $p_{2} s_{1}$ & 1.41 & 2851.90 & 0.00 & 10 & 42.96 & 2851.90 & 0.00 & 10 \\
\hline 100 & 20 & $p_{2} s_{2}$ & 1800.00 & 1635.40 & 0.53 & 0 & 1800.00 & 1635.40 & 0.65 & 0 \\
\hline 100 & 20 & $p_{2} s_{3}$ & 0.05 & 3848.50 & 0.00 & 10 & 0.08 & 3848.50 & 0.00 & 10 \\
\hline 500 & 20 & $p_{2} s_{1}$ & 515.57 & 69545.40 & 0.02 & 8 & 895.87 & 69536.40 & 0.01 & 6 \\
\hline 500 & 20 & $p_{2} s_{2}$ & 1800.00 & 39087.00 & 2.20 & 0 & 1800.00 & 38577.40 & 0.92 & 0 \\
\hline 500 & 20 & $p_{2} s_{3}$ & 185.12 & 91522.60 & 0.001 & 9 & 192.42 & 91522.90 & 0.002 & 9 \\
\hline 1000 & 20 & $p_{2} s_{1}$ & 1800.00 & 271969.40 & 0.05 & 0 & 1402.05 & 271888.10 & 0.01 & 3 \\
\hline 1000 & 20 & $p_{2} s_{2}$ & 1800.00 & 501425.80 & 100.67 & 0 & 1800.00 & 155898.30 & 3.47 & 0 \\
\hline 1000 & 20 & $p_{2} s_{3}$ & 15.01 & 368942.80 & 0.00 & 10 & 99.63 & 368942.80 & 0.00 & 10 \\
\hline 5000 & 20 & $p_{2} s_{1}$ & 1800.00 & 12434970.20 & 507.61 & 0 & 1800.00 & 6624330.30 & 0.05 & 0 \\
\hline 5000 & 20 & $p_{2} s_{2}$ & 1800.00 & 12525365.00 & 99.96 & 0 & 1800.00 & - & & $0(2)$ \\
\hline 5000 & 20 & $p_{2} s_{3}$ & 1718.22 & 9063247.70 & 0.001 & 1 & 1656.91 & 9063471.90 & 0.004 & 1 \\
\hline \multicolumn{3}{|c|}{ Avg./Sum. } & 739.52 & 1965367.85 & 39.50 & 108 & 747.20 & - & & 109 \\
\hline
\end{tabular}

Table 10: Computational results for new instances proposed $(B=20)$. 


\begin{tabular}{|c|c|c|c|c|c|c|c|c|c|c|}
\hline \multirow{2}{*}{ Jobs } & \multirow{2}{*}{$\begin{array}{l}\text { Instance } \\
\text { Capacity }\end{array}$} & \multirow{2}{*}{ Type } & \multicolumn{4}{|c|}{$\left(\mathrm{MILP}_{1}^{+}\right)$} & \multicolumn{4}{|c|}{$(\mathrm{FLOW})$} \\
\hline & & & $T(s)$ & $C_{\max }$ & Gap & $\# \mathrm{O}$ & $T(s)$ & $C_{\max }$ & Gap & $\# \mathrm{O}$ \\
\hline \multicolumn{11}{|c|}{ Instances with $p_{1}=[1,20]$ and $B=50$} \\
\hline 10 & 50 & $p_{1} s_{1}$ & 0.01 & 62.10 & 0.00 & 10 & 0.09 & 62.10 & 0.00 & 10 \\
\hline 10 & 50 & $p_{1} s_{2}$ & 0.01 & 40.40 & 0.00 & 10 & 0.16 & 40.40 & 0.00 & 10 \\
\hline 10 & 50 & $p_{1} s_{3}$ & 0.00 & 95.90 & 0.00 & 10 & 0.01 & 95.90 & 0.00 & 10 \\
\hline 50 & 50 & $p_{1} s_{1}$ & 0.16 & 300.60 & 0.00 & 10 & 17.74 & 300.60 & 0.00 & 10 \\
\hline 50 & 50 & $p_{1} s_{2}$ & 62.53 & 168.90 & 0.00 & 10 & 58.47 & 168.90 & 0.00 & 10 \\
\hline 50 & 50 & $p_{1} s_{3}$ & 0.01 & 437.50 & 0.00 & 10 & 0.04 & 437 & 0.00 & 10 \\
\hline 100 & 50 & $p_{1} s_{1}$ & 0.53 & 579.80 & 0.00 & 10 & 9.64 & 579.80 & 0.00 & 10 \\
\hline 100 & 50 & $p_{1} s_{2}$ & 1800.00 & 322.00 & 0.91 & 0 & 935.07 & 321.90 & 0.16 & 7 \\
\hline 100 & 50 & $p_{1} s_{3}$ & 0.03 & 795.50 & 0.00 & 10 & 0.06 & & 0.00 & 10 \\
\hline 500 & 50 & $p_{1} s_{1}$ & 994.13 & 2789.50 & 0.05 & 6 & 78.06 & 2789.00 & 0.00 & 10 \\
\hline 500 & 50 & $p_{1} s_{2}$ & 1800.00 & 1610.50 & 2.60 & 0 & 1599.21 & 1584.70 & 0.48 & 2 \\
\hline 500 & 50 & $p_{1} s_{3}$ & 0.96 & 3981.00 & 0.00 & 10 & 0.11 & 3981.00 & 0.00 & 10 \\
\hline 1000 & 50 & $p_{1} s_{1}$ & 1724.32 & 5511.30 & 0.14 & 3 & 196.69 & 5507.90 & 0.003 & 9 \\
\hline 1000 & 50 & $p_{1} s_{2}$ & 1800.00 & 10430.30 & 114.18 & 0 & 1670.09 & 3138.50 & 0.23 & $J$ \\
\hline 1000 & 50 & $p_{1} s_{3}$ & 86.26 & 7865.40 & 0.00 & 10 & 0.59 & 7865.40 & 0.00 & 10 \\
\hline 5000 & 50 & $p_{1} s_{1}$ & 1800.00 & 52466.40 & 183.12 & 0 & 32.02 & 27157.30 & 0.00 & 10 \\
\hline 5000 & 50 & $p_{1} s_{2}$ & 1800.00 & 52469.40 & 99.96 & 0 & 1583.74 & 15745.70 & 0.05 & 3 \\
\hline 5000 & 50 & $p_{1} s_{3}$ & 1426.51 & 38976.90 & 0.02 & 4 & 0.89 & 38971.20 & 0.00 & 10 \\
\hline \multicolumn{3}{|c|}{ Avg./Sum. } & 738.64 & 9939.08 & 22.28 & 113 & 343.48 & 6085.74 & 0.05 & 154 \\
\hline \multicolumn{11}{|c|}{ Instances with $p_{2}=\left[1, n_{J}\right]$ and $B=50$} \\
\hline 10 & 50 & $p_{2} s_{1}$ & 0.01 & 39.70 & 0.00 & 10 & 0.04 & & 0.00 & 10 \\
\hline 10 & 50 & $p_{2} s_{2}$ & 0.01 & 23.00 & 0.00 & 10 & 0.14 & 23.00 & 0.00 & 10 \\
\hline 10 & 50 & $p_{2} s_{3}$ & 0.00 & 46.90 & 0.00 & 10 & 0.01 & 46.90 & 0.00 & 10 \\
\hline 50 & 50 & $p_{2} s_{1}$ & 0.16 & 733.90 & 0.00 & 10 & 29.05 & 733.90 & 0.00 & 10 \\
\hline 50 & 50 & $p_{2} s_{2}$ & 112.30 & 401.60 & 0.00 & 10 & 801.15 & 401.70 & 0.32 & 7 \\
\hline 50 & 50 & $p_{2} s_{3}$ & 0.01 & 965.20 & 0.00 & 10 & 0.07 & 965.20 & 0.00 & 10 \\
\hline 100 & 50 & $p_{2} s_{1}$ & 1.71 & 2684.50 & 0.00 & 10 & 685.70 & 2684.60 & 0.06 & 7 \\
\hline 100 & 50 & $p_{2} s_{2}$ & 1653.76 & 1608.40 & 0.48 & 2 & 1800.00 & 1612.80 & 1.29 & 0 \\
\hline 100 & 50 & $p_{2} s_{3}$ & 0.03 & 4138.30 & 0.00 & 10 & 0.14 & 4138.30 & 0.00 & 10 \\
\hline 500 & 50 & $p_{2} s_{1}$ & 660.84 & 66528.90 & 0.03 & 8 & 1600.57 & 66549.40 & 0.08 & $3 \quad 2$ \\
\hline 500 & 50 & $p_{2} s_{2}$ & 1800.00 & 38883.80 & 2.73 & 0 & 1800.00 & - & & $-0(9)$ \\
\hline 500 & 50 & $p_{2} s_{3}$ & 0.83 & 94950.00 & 0.00 & 10 & 14.81 & 94950.00 & 0.00 & 10 \\
\hline 1000 & 50 & $p_{2} s_{1}$ & 1675.45 & 264095.80 & 0.17 & 2 & 1731.41 & - & & $-1(9)$ \\
\hline 1000 & 50 & $p_{2} s_{2}$ & 1800.00 & 467387.20 & 90.86 & 0 & 1800.00 & - & & $-0(1)$ \\
\hline 1000 & 50 & $p_{2} s_{3}$ & 186.15 & 370468.90 & 0.0003 & 9 & 208.64 & 370468.90 & 0.001 & 9 \\
\hline 5000 & 50 & $p_{2} s_{1}$ & 1800.00 & 12541306.50 & 163.00 & 0 & 1800.00 & - & & $-0(0)$ \\
\hline 5000 & 50 & $p_{2} s_{2}$ & 1800.00 & 12571888.70 & 99.96 & 0 & 1800.00 & - & & $-0(0)$ \\
\hline 5000 & 50 & $p_{2} s_{3}$ & 678.76 & 9288025.00 & 0.0001 & 8 & 874.70 & 9288030.00 & 0.0003 & 6 \\
\hline \multicolumn{3}{|c|}{ Avg./Sum. } & 676.11 & 1984120.91 & 19.85 & 119 & 830.36 & - & - & -102 \\
\hline
\end{tabular}

Table 11: Computational results for new instances proposed $(B=50)$. 


\begin{tabular}{|c|c|c|c|c|c|c|c|c|c|c|}
\hline \multirow{2}{*}{\multicolumn{3}{|c|}{$\begin{array}{c}\text { Instance } \\
\text { Jobs Capacity Type }\end{array}$}} & \multicolumn{4}{|c|}{$\left(\mathrm{MILP}_{1}^{+}\right)$} & \multicolumn{4}{|c|}{ (FLOW) } \\
\hline & & & $T(s)$ & $C_{\max }$ & Gap & $\# \mathrm{O}$ & $T(s)$ & $C_{\max }$ & Gap & $\# \mathrm{O}$ \\
\hline \multicolumn{11}{|c|}{ Instances with $p_{1}=[1,20]$ and $B=100$} \\
\hline 10 & 100 & $p_{1} s_{1}$ & 0.01 & 66.40 & 0.00 & 10 & 0.15 & 66.40 & 0.00 & 10 \\
\hline 10 & 100 & $p_{1} s_{2}$ & 0.02 & 45.10 & 0.00 & 10 & 0.26 & 45.10 & 0.00 & 10 \\
\hline 10 & 100 & $p_{1} s_{3}$ & 0.00 & 96.00 & 0.00 & 10 & 0.01 & 96.00 & 0.00 & 10 \\
\hline 50 & 100 & $p_{1} s_{1}$ & 0.08 & 306.10 & 0.00 & 10 & 10.90 & 306.10 & 0.00 & 10 \\
\hline 50 & 100 & $p_{1} s_{2}$ & 15.08 & 174.30 & 0.00 & 10 & 212.80 & 174.30 & 0.00 & 10 \\
\hline 50 & 100 & $p_{1} s_{3}$ & 0.01 & 399.8 & 0.00 & 10 & 0.06 & 399 & 0.00 & 10 \\
\hline 100 & 100 & $p_{1} s_{1}$ & 1.60 & 583.20 & 0.00 & 10 & 161.24 & 583 & 0.00 & 10 \\
\hline 100 & 100 & $p_{1} s_{2}$ & 1540.99 & 327.70 & 0.76 & 2 & 1588.46 & 327.90 & 0.76 & 3 \\
\hline 100 & 100 & $p_{1} s_{3}$ & 0.03 & 825.80 & 0.00 & 10 & 0.13 & 825.80 & 0.00 & 10 \\
\hline 500 & 100 & $p_{1} s_{1}$ & 785.67 & 2773.40 & 0.04 & 6 & 601.28 & 2773.00 & 0.01 & 8 \\
\hline 500 & 100 & $p_{1} s_{2}$ & 1800.00 & 1614.80 & 3.46 & 0 & 1800.00 & & 2.23 & 0 \\
\hline 500 & 100 & $p_{1} s_{3}$ & 0.98 & 4051.20 & 0.00 & 10 & 0.65 & 4051.20 & 0.00 & 10 \\
\hline 1000 & 100 & $p_{1} s_{1}$ & 1800.00 & & 0.49 & 0 & 793.25 & 5540.60 & 0.01 & 7 \\
\hline 1000 & 100 & $p_{1} s_{2}$ & 1800.00 & 10493.40 & 111.21 & 0 & 1800.00 & 3202.00 & 1.90 & 0 \\
\hline 1000 & 100 & $p_{1} s_{3}$ & 182.03 & & 0.00 & 10 & 1.25 & 7948.70 & 0.00 & 10 \\
\hline 5000 & 100 & $p_{1} s_{1}$ & 1800.00 & 52385.90 & 131.52 & 0 & 942.72 & 26798.70 & 0.001 & 8 \\
\hline 5000 & 100 & $p_{1} s_{2}$ & 1800.00 & 52390.60 & 99.96 & 0 & 1800.00 & 15849.00 & 0.69 & 0 \\
\hline 5000 & 100 & $p_{1} s_{3}$ & 576.32 & 39365.10 & 0.00 & 10 & 0.58 & 39365.10 & 0.00 & 10 \\
\hline \multicolumn{3}{|c|}{ Avg./Sum. } & 672.38 & 9966.83 & 19.30 & 118 & | 539.65 & 6108.52 & 0.31 & 136 \\
\hline \multicolumn{11}{|c|}{ Instances with $p_{2}=\left[1, n_{J}\right]$ and $B=100$} \\
\hline 10 & 100 & $p_{2} s_{1}$ & 0.01 & 32.10 & 0.00 & 10 & 0.15 & 32.10 & 0.00 & 10 \\
\hline 10 & 100 & $p_{2} s_{2}$ & 0.02 & 20.20 & 0.00 & 10 & 0.26 & 20.20 & 0.00 & 10 \\
\hline 10 & 100 & $p_{2} s_{3}$ & 0.00 & 48.30 & 0.00 & 10 & 0.01 & 48.30 & 0.00 & 10 \\
\hline 50 & 100 & $p_{2} s_{1}$ & 0.13 & 778.40 & 0.00 & 10 & 275.12 & 778.40 & 0.06 & 9 \\
\hline 50 & 100 & $p_{2} s_{2}$ & 33.16 & 425.00 & 0.00 & 10 & 1287.58 & 425.10 & 0.63 & 6 \\
\hline 50 & 100 & $p_{2} s_{3}$ & 0.01 & 1033.90 & 0.00 & 10 & 0.11 & 1033.90 & 0.00 & 10 \\
\hline 100 & 100 & $p_{2} s_{1}$ & 1.81 & 2954.60 & 0.00 & 10 & 609.02 & 2955.80 & 0.21 & 8 \\
\hline 100 & 100 & $p_{2} s_{2}$ & 1800.00 & 1608.70 & 0.50 & 0 & 1800.00 & 1619.10 & 1.97 & 0 \\
\hline 100 & 100 & $p_{2} s_{3}$ & 0.03 & 3977.90 & 0.00 & 10 & 0.61 & 3977.90 & 0.00 & 10 \\
\hline 500 & 100 & $p_{2} s_{1}$ & 982.89 & 66060.70 & 0.02 & 5 & 1800.00 & - & & $0(6)$ \\
\hline 500 & 100 & $p_{2} s_{2}$ & 1800.00 & 39288.80 & 3.71 & 0 & 1800.00 & - & & $0(8)$ \\
\hline 500 & 100 & $p_{2} s_{3}$ & 0.79 & 97723.40 & 0.00 & 10 & 187.89 & 97723.40 & 0.0004 & 9 \\
\hline 1000 & 100 & $p_{2} s_{1}$ & 1800.00 & 261987.60 & 0.40 & 0 & 1800.00 & - & & $0(0)$ \\
\hline 1000 & 100 & $p_{2} s_{2}$ & 1800.00 & 502509.30 & 100.36 & 0 & 1800.00 & 181386.00 & 15.86 & 0 \\
\hline 1000 & 100 & $p_{2} s_{3}$ & 4.90 & 384407.30 & 0.00 & 10 & 77.20 & 384407.30 & 0.00 & 10 \\
\hline 5000 & 100 & $p_{2} s_{1}$ & 1800.00 & 12564195.20 & 110.74 & 0 & 1800.00 & - & & $0(0)$ \\
\hline 5000 & 100 & $p_{2} s_{2}$ & 1800.00 & 12468660.50 & 99.96 & 0 & 1800.00 & - & - & $0(0)$ \\
\hline 5000 & 100 & $p_{2} s_{3}$ & 624.90 & 9408522.00 & 0.00002 & 9 & 1387.97 & 9408543.80 & 0.001 & 3 \\
\hline \multicolumn{3}{|c|}{ Avg./Sum. } & 691.59 & 1989124.11 & 17.54 & 114 & 912.55 & - & - & 95 \\
\hline
\end{tabular}

Table 12: Computational results for new instances proposed $(B=100)$.

(C) 2021. This manuscript version is made available under the CC BY-NC-ND 4.0 license. 


\begin{tabular}{|c|c|c|c|c|c|c|c|c|c|}
\hline \multirow{2}{*}{ Jobs } & \multirow{2}{*}{$\begin{array}{l}\text { Instance } \\
\text { Capacity }\end{array}$} & \multirow{2}{*}{ Type } & \multicolumn{4}{|c|}{$\left(\mathrm{MILP}_{1}^{+}\right)$} & \multicolumn{3}{|c|}{$(\mathrm{FLOW})$} \\
\hline & & & $T(s)$ & $C_{\max }$ & Gap & $\# \mathrm{O}$ & $T(s)$ & $C_{\max }$ & Gap \#O \\
\hline 10 & 500 & $p_{1} s_{1}$ & 0.01 & 69.7 & 0.00 & 10 & 0.24 & 69.70 & 0.00 \\
\hline 50 & 500 & & & 287.00 & 0.00 & 10 & 490.97 & 287.10 & 0.12 \\
\hline 100 & 500 & $p_{1} s_{1}$ & & 575.80 & 0.00 & 10 & 1169.57 & 577.30 & 0.53 \\
\hline 500 & 500 & $p_{1} s_{1}$ & 1118. & 2708.60 & 0.05 & 7 & 1800.00 & - & $-0(1)$ \\
\hline 1000 & 500 & $p_{1} s_{1}$ & & 5414.20 & 1.03 & 0 & 1800.00 & - & $-0(0)$ \\
\hline 5000 & 500 & $p_{1} s_{1}$ & 1800.00 & 52439.90 & 110.63 & 0 & 1800.00 & - & $-0(0)$ \\
\hline \multicolumn{3}{|c|}{ Avg./Sum. } & 787.07 & 10249.20 & 18.62 & 37 & $\mid 1176.80$ & & 23 \\
\hline 10 & 500 & $p_{2} s_{1}$ & & & 0.00 & 10 & 0.47 & 33.60 & 0.00 \\
\hline 50 & 500 & & & 710.80 & 0.00 & 10 & 436.26 & 710.80 & $0.11 \quad 9$ \\
\hline 100 & 500 & $p_{2} s_{1}$ & & 2616.60 & 0.00 & 10 & 1641.37 & & $-4(8)$ \\
\hline 500 & 500 & $p_{2} s_{1}$ & 932.9 & 63734.80 & 0.04 & 6 & 1800.00 & & $-0(0)$ \\
\hline 1000 & 500 & $p_{2} s_{1}$ & 1800.00 & 260454.00 & 0.57 & 0 & 1800.00 & & $-0(0)$ \\
\hline 5000 & 500 & $p_{2} s_{1}$ & 1800.00 & 12553378.80 & 100.67 & 0 & 1800.00 & - & $-0(0)$ \\
\hline \multicolumn{3}{|c|}{ Avg./Sum. } & 755.66 & 2146821.43 & 16.88 & 36 & | 1246.35 & - & 23 \\
\hline
\end{tabular}

Table 13: Computational results for new instances proposed $(B=500)$.

\begin{tabular}{|c|c|c|c|c|c|c|c|c|c|c|}
\hline & \multirow{2}{*}{$\begin{array}{l}\text { Instance } \\
\text { Capacity }\end{array}$} & \multirow[b]{2}{*}{ Type } & \multicolumn{4}{|c|}{$\left(\mathrm{MILP}_{1}^{+}\right)$} & \multicolumn{4}{|c|}{ (FLOW) } \\
\hline & & & $T(s)$ & $C_{\max }$ & Gap & $\# \mathrm{O}$ & $T(s)$ & $C_{\max }$ & Gap & $\# \mathrm{O}$ \\
\hline 10 & 1000 & $p_{1} s_{1}$ & 0.00 & 65.90 & 0.00 & 10 & 0.89 & 65.90 & 0.00 & 10 \\
\hline 50 & 00 & $p_{1} s_{1}$ & & 277.90 & 0.00 & 10 & 698.14 & 278.10 & 0.57 & 8 \\
\hline 100 & 1000 & $p_{1} s_{1}$ & & 56 & 0.00 & 10 & 1535.94 & 572.90 & 1.24 & 4 \\
\hline 500 & 000 & & 986.90 & 2683.60 & 0.02 & 8 & 1800.00 & - & & $0(1)$ \\
\hline 1000 & 000 & $p_{1} s_{1}$ & 180 & 549 & 0.73 & 0 & 1800.00 & - & & $0(0)$ \\
\hline 5000 & 1000 & $p_{1} s_{1}$ & 1800.00 & 52521.00 & 109.44 & 0 & 1800.00 & - & & $0(0)$ \\
\hline \multicolumn{3}{|c|}{ Avg./Sum. } & 765.58 & 10267.90 & 18.37 & 38 & $\mid 1272.50$ & - & - & 22 \\
\hline 10 & 1000 & $p_{2} s_{1}$ & 0.01 & 37.20 & 0.00 & 10 & 0.35 & 37.20 & 0.00 & 10 \\
\hline 50 & 1000 & $p_{2} s_{1}$ & 0.16 & 769.40 & 0.00 & 10 & 634.07 & 769.60 & 0.20 & 8 \\
\hline 100 & 1000 & $p_{2} s_{1}$ & 0.59 & 2861.00 & 0.00 & 10 & 1699.17 & - & & $2(6)$ \\
\hline 500 & 1000 & $p_{2} s_{1}$ & 1106.21 & 65039.50 & 0.03 & 5 & 1800.00 & - & & $0(0)$ \\
\hline 1000 & & $p_{2} s_{1}$ & 1800.00 & 258876.10 & 0.76 & 0 & 1800.00 & - & & $0(0)$ \\
\hline 5000 & 1000 & $p_{2} s_{1}$ & 1800.00 & 12495215.80 & 100.46 & 0 & 1800.00 & - & & $0(0)$ \\
\hline \multicolumn{3}{|c|}{ Avg./Sum. } & 784.49 & 2137133.17 & 16.87 & 35 & | 1288.93 & - & - & 20 \\
\hline
\end{tabular}

Table 14: Computational results for new instances proposed $B=1000$ ). 
Analyzing the parameters that affect the number of variables and constraints in our arc-flow formulation, we conclude that the formulation is very effective for instances of the problem where, although the number of jobs is very large, they have similar characteristics, so the numbers of distinct processing times and job sizes are not very large. In order to investigate the scability of our approach, we have generated a new set of instances where the parameters setting had the purpose of making the instances more challenging for the model than the benchmark instances from the literature. We see that our model complements another model from the literature, in the sense that depending on the characteristics of the instances, each one can perform better than the other. These characteristics are identified and can be found on different applications for the problem. For example, in burn-in tests for semiconductors, it is natural to have many jobs to perform with similar characteristics of processing times and sizes. These tests were mentioned in the literature as an important application for the single batch-processing machine scheduling problem.

As future research, we would like to investigate if the good performance of the formulation presented can be replicated when the arc-flow based approach is applied to other problems in the vast area of scheduling applications as, for example, scheduling a batch-processing machine with incompatible job families and single and parallel batch-processing machine scheduling with arbitrary release times.

\section{Acknowledgments}

R. S. Trindade was partially supported by a Ph.D. scholarship from the Brazilian National Council for Scientific and Technological Development (CNPq), grant 142205/2014-1, and by CNPq grant 303898/2016-0. M. Fampa was supported in part by CNPq grants 303898/2016-0 and 434683/20183. The authors thank [4] and [29] for supplying them with their set of test instances. We thank the anonymous referees for their suggestions and helpful comments, which have significantly improved the presentation of this paper.

\section{References}

[1] Muhammad Al-Salamah. Constrained binary artificial bee colony to minimize the makespan for single machine batch processing with non-identical job sizes. Applied Soft Computing, 29:379385 , apr 2015.

[2] Filipe Brandão and João Pedro Pedroso. Bin packing and related problems: General arc-flow formulation with graph compression. Computers \& Operations Research, 69:56-67, may 2016.

[3] Filipe Daniel Alves Brandão. Cutting 8 Packing Problems: General Arc-flow Formulation with Graph Compression. PhD thesis, Universidade do Porto, 2017.

[4] Huaping Chen, Bing Du, and George Q. Huang. Scheduling a batch processing machine with non-identical job sizes: a clustering perspective. International Journal of Production Research, 49(19):5755-5778, oct 2011.

[5] S.H. Chung, Y.T. Tai, and W.L. Pearn. Minimising makespan on parallel batch processing machines with non-identical ready time and arbitrary job sizes. International Journal of Production Research, 47(18):5109-5128, sep 2009.

[6] Jean François Côté and Manuel Iori. The meet-in-the-middle principle for cutting and packing problems. INFORMS Journal on Computing, 30(4):646-661, 2018.

[7] Purushothaman Damodaran, Omar Ghrayeb, and Mallika Chowdary Guttikonda. GRASP to minimize makespan for a capacitated batch-processing machine. The International Journal of Advanced Manufacturing Technology, 68(1-4):407-414, jan 2013.

(C) 2021. This manuscript version is made available under the CC BY-NC-ND 4.0 license. 
[8] Purushothaman Damodaran, Praveen Kumar Manjeshwar, and Krishnaswami Srihari. Minimizing makespan on a batch-processing machine with non-identical job sizes using genetic algorithms. International Journal of Production Economics, 103(2):882-891, oct 2006.

[9] Vinícius L. de Lima, Cláudio Alves, François Clautiaux, Manuel Iori, and José M. Valério de Carvalho. Arc flow formulations based on dynamic programming: Theoretical foundations and applications. European Journal of Operational Research, 2021.

[10] Maxence Delorme, Manuel Iori, and Silvano Martello. Bin packing and cutting stock problems: Mathematical models and exact algorithms. European Journal of Operational Research, 255(1):1-20, nov 2016.

[11] Lionel Dupont and Clarisse Dhaenens-Flipo. Minimizing the makespan on a batch machine with non-identical job sizes: an exact procedure. Computers $\&$ Operations Research, 29(7):807-819, 2002 .

[12] Lionel Dupont and Fariborz Jolai Ghazvini. Minimizing makespan on a single batch processing machine with non-identical job sizes. European Journal of Automation Systems, 32:431-40, 1998.

[13] Fariborz Jolai Ghazvini and Lionel Dupont. Minimizing mean flow times criteria on a single batch processing machine with non-identical jobs sizes. International Journal of Production Economics, 55:273-280, aug 1998.

[14] Zhao-hong Jia and Joseph Y.-T. Leung. An improved meta-heuristic for makespan minimization of a single batch machine with non-identical job sizes. Computers $\&$ Operations Research, 46:4958 , jun 2014 .

[15] a. H. Kashan, B. Karimi, and F. Jolai. Effective hybrid genetic algorithm for minimizing makespan on a single-batch-processing machine with non-identical job sizes. International Journal of Production Research, 44(12):2337-2360, jun 2006.

[16] Arthur Kramer, Mauro Dell'Amico, Dominique Feillet, and Manuel Iori. Scheduling jobs with release dates on identical parallel machines by minimizing the total weighted completion time. Computers $\&$ Operations Research, 123:105018, 2020.

[17] Arthur Kramer, Mauro Dell'Amico, and Manuel Iori. Enhanced arc-flow formulations to minimize weighted completion time on identical parallel machines. European Journal of Operational Research, 275(1):67-79, may 2019.

[18] Arthur Kramer, Manuel Iori, and Philippe Lacomme. Mathematical formulations for scheduling jobs on identical parallel machines with family setup times and total weighted completion time minimization. European Journal of Operational Research, 289(3):825-840, 2021.

[19] Chung-Yee Lee, Reha Uzsoy, and Louis A Martin-Vega. Efficient Algorithms for Scheduling Semiconductor Burn-In Operations. Operations Research, 40(4):pp. 764-775, aug 1992.

[20] Yoon Ho Lee and Young Hoon Lee. Minimising makespan heuristics for scheduling a single batch machine processing machine with non-identical job sizes. International Journal of Production Research, 51(12):3488-3500, jun 2013.

[21] Xiao Lin Li, Yu Peng Li, and Yu Wang. Minimising makespan on a batch processing machine using heuristics improved by an enumeration scheme. International Journal of Production Research, 55(1):176-186, jun 2017.

[22] Rita Macedo, Cláudio Alves, and J.M. Valério de Carvalho. Arc-flow model for the twodimensional guillotine cutting stock problem. Computers \& Operations Research, 37(6):991 $-1001,2010$.

(C) 2021. This manuscript version is made available under the CC BY-NC-ND 4.0 license. 
[23] J. Martinovic, G. Scheithauer, and J. M. Valério de Carvalho. A comparative study of the arcflow model and the one-cut model for one-dimensional cutting stock problems. European Journal of Operational Research, 266(2):458-471, apr 2018.

[24] M. Mathirajan and A. Sivakumar. A literature review, classification and simple meta-analysis on scheduling of batch processors in semiconductor. The International Journal of Advanced Manufacturing Technology, 29(9-10):990-1001, jan 2006.

[25] Sharif Melouk, Purushothaman Damodaran, and Ping-Yu Chang. Minimizing makespan for single machine batch processing with non-identical job sizes using simulated annealing. International Journal of Production Economics, 87(2):141-147, jan 2004.

[26] Ying Meng and Lixin Tang. A tabu search heuristic to solve the scheduling problem for a batch-processing machine with non-identical job sizes. In 2010 International Conference on Logistics Systems and Intelligent Management (ICLSIM), volume 3, pages 1703-1707. IEEE, jan 2010.

[27] Lars Mönch, John W. Fowler, Stéphane Dauzère-Pérès, Scott J. Mason, and Oliver Rose. A survey of problems, solution techniques, and future challenges in scheduling semiconductor manufacturing operations. Journal of Scheduling, 14(6):583-599, jan 2011.

[28] Mehdi Mrad and Nizar Souayah. An Arc-Flow Model for the Makespan Minimization Problem on Identical Parallel Machines. IEEE Access, 6:5300-5307, jan 2018.

[29] Ibrahim Muter. Exact Algorithms to Minimize Makespan on Single and Parallel Batch Processing Machines. European Journal of Operational Research, feb 2020.

[30] A. Oulamara, G. Finke, and A. Kamgaing Kuiteing. Flowshop scheduling problem with a batching machine and task compatibilities. Computers $\&$ Operations Research, 36(2):391-401, feb 2009.

[31] Onur Ozturk, Mehmet A. Begen, and Gregory S. Zaric. A branch and bound based heuristic for makespan minimization of washing operations in hospital sterilization services. European Journal of Operational Research, 239(1):214-226, nov 2014.

[32] Chris N. Potts and Mikhail Y. Kovalyov. Scheduling with batching: A review. European Journal of Operational Research, 120(2):228-249, jan 2000.

[33] N. Rafiee Parsa, B. Karimi, and A. Husseinzadeh Kashan. A branch and price algorithm to minimize makespan on a single batch processing machine with non-identical job sizes. Computers G Operations Research, 37(10):1720-1730, oct 2010.

[34] Y.T. Tai. The Study on the Production Scheduling Problems for Liquid Crystal Display Module Assembly factories. PhD thesis, National Chiao Tung University, jul 2008.

[35] Renan Spencer Trindade. Modelling Batch Processing Machines Problems with Symmetry Breaking and Arc Flow Formulation. PhD thesis, Universidade Federal do Rio de Janeiro, 2019.

[36] Renan Spencer Trindade, Olinto C.B. de Araújo, and Marcia Fampa. Arc-flow approach for parallel batch processing machine scheduling with non-identical job sizes. In Mahjoub A.R. Baïou M., Gendron B., Günlük O., editor, Combinatorial Optimization, pages 179-190. Springer International Publishing, 2020.

[37] Renan Spencer Trindade, Olinto César Bassi de Araújo, Marcia Fampa, and Felipe Martins Müller. Modelling and symmetry breaking in scheduling problems on batch processing machines. International Journal of Production Research, 56(22):7031-7048, nov 2018.

[38] R. Uzsoy. Scheduling a single batch processing machine with non-identical job sizes. International Journal of Production Research, 32(7):1615-1635, jul 1994.

(C) 2021. This manuscript version is made available under the CC BY-NC-ND 4.0 license. 
[39] J.M. Valério de Carvalho. Exact solution of cutting stock problems using column generation and branch-and-bound. International Transactions in Operational Research, 5(1):35-44, 1998.

[40] J.M. Valério de Carvalho. Exact solution of bin-packing problems using column generation and branch-and-bound. Annals of Operations Research, 86:629-659, jan 1999.

[41] François Vanderbeck. Computational study of a column generation algorithm for bin packing and cutting stock problems. Mathematical Programming, 86(3):565-594, dec 1999.

[42] Guochuan Zhang, Xiaoqiang Cai, C.-Y Lee, and C.K Wong. Minimizing makespan on a single batch processing machine with nonidentical job sizes. Naval Research Logistics, 48(3):226-240, apr 2001.

[43] Nihat Öner, Hakan Gultekin, and Çăgrı Koç. The airport shuttle bus scheduling problem. International Journal of Production Research, 0(0):1-23, 2020.

(C) 2021. This manuscript version is made available under the CC BY-NC-ND 4.0 license. 